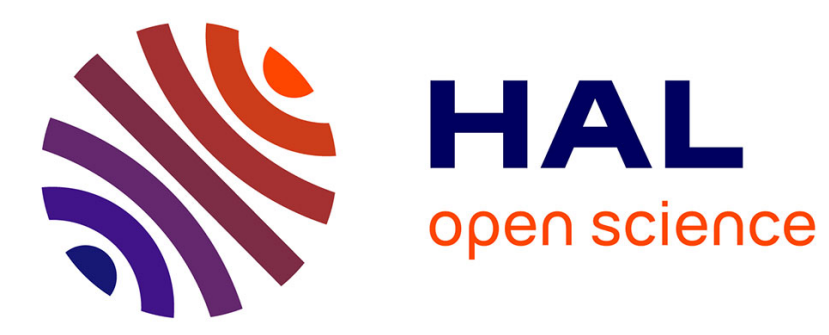

\title{
The Lamé family of connections on the projective line
} Frank Loray, Marius van Der Put, Félix Ulmer

\section{To cite this version:}

Frank Loray, Marius van Der Put, Félix Ulmer. The Lamé family of connections on the projective line. Annales de la Faculté des Sciences de Toulouse. Mathématiques., 2008, 17, pp.371-409. hal00005796v2

\section{HAL Id: hal-00005796 \\ https://hal.science/hal-00005796v2}

Submitted on 28 Mar 2006

HAL is a multi-disciplinary open access archive for the deposit and dissemination of scientific research documents, whether they are published or not. The documents may come from teaching and research institutions in France or abroad, or from public or private research centers.
L'archive ouverte pluridisciplinaire HAL, est destinée au dépôt et à la diffusion de documents scientifiques de niveau recherche, publiés ou non, émanant des établissements d'enseignement et de recherche français ou étrangers, des laboratoires publics ou privés. 


\title{
THE LAMÉ FAMILY OF CONNECTIONS ON THE PROJECTIVE LINE
}

\author{
F. LORAY, M. VAN DER PUT AND F. ULMER
}

\begin{abstract}
This paper deals with rank two connections on the projective line having four simple poles with prescribed local exponents $1 / 4$ and $-1 / 4$. This Lamé family of connections has been extensively studied in the literature. The differential Galois group of a Lamé connection is never maximal: it is either dihedral (finite or infinite) or reducible. We provide an explicit moduli space of those connections having a free underlying vector bundle and compute the algebraic locus of those reducible connections. The irreducible Lamé connections are derived from the rank 1 regular connections on the elliptic curve $w^{2}=z(z-1)(z-t)$; those connections having a finite Galois group are known to be related to points of finite order on the elliptic curve. In the paper, we provide a very efficient algorithm to compute the locus of those Lamé connections having a finite Galois group of a given order. We also give an efficient algorithm to compute the minimal polynomial for the corresponding field extension. We do this computation for low order and recover this way known algebraic solutions of the Painlevé VI equation and of the classical Lamé equation. In the final section we compare our moduli space with the classical one due to Okamoto.
\end{abstract}

\section{INTRODUCTION}

A Lamé connection is a rank two connection $(\mathcal{M}, \nabla)$ on the projective line having simple poles at $0,1, t$ and $\infty$, with local exponents $1 / 4$ and $-1 / 4$ at each of those singular points. This family of connections naturally arises from several points of view.

First of all, the Galois group of such a connection is very special: it is either dihedral, or reducible. This is due to the fact that, all irreducible Lamé connections are derived by pushing forward a regular rank 1 connection on the Legendre elliptic curve $E_{t}: w^{2}=z(z-1)(z-t)$ via the 2 -fold cover $(z, w) \mapsto z$. In particular, the monodromy representation can be explicitely computed by means of elliptic functions.

Date: March, 2006.

1991 Mathematics Subject Classification. 12H05, 14D20, 34A30, 68W30. 
These facts were observed by N. Hitchin in [7]; another proof has been communicated to us by F. Beukers ([3]).

Beside being dihedral, the Galois group of an irreducible Lamé connection may be finite dihedral $D_{N}^{S L_{2}}$ or infinite dihedral $D_{\infty}^{S L_{2}}$ (these groups are the unique liftings of the classical projective dihedral groups $D_{N}$ and $D_{\infty}$ under the map $\mathrm{SL}_{2} \rightarrow \mathrm{PSL}_{2}$, i.e. the order 2 central extensions). It turns out that all $D_{N}^{S L_{2}}$ actually occur inside the family. The goal of our paper is mainly to produce an efficient algorithm computing the locus of those connections having finite Galois group $D_{N}^{S L_{2}}$. Each $D_{N}^{S L_{2}}$ corresponds to a closed, codimension 2, subset of the Lamé family, showing that the locus of $D_{\infty}^{S L_{2}}$ is non constructible. This fact was already observed by M.F. Singer in [22] (see also [2]) and the Lamé family is the simplest family of connections for which this phenomenon occurs.

The underlying vector bundle of a Lamé connection can be of two types: either it is free, the trivial vector bundle, or it is $\mathcal{O}(1) \oplus \mathcal{O}(-1)$. We provide in Section 5 an algebraic construction of the moduli space for those Lamé connections having free underlying vector bundle, that is for Lamé Fuchsian systems:

$$
\frac{d}{d z}+\frac{A_{1}}{z}+\frac{A_{2}}{z-1}+\frac{A_{3}}{z-t} \quad \text { with } \quad A_{j}=\left(\begin{array}{cc}
a_{j} & b_{j} \\
c_{j} & -a_{j}
\end{array}\right)
$$

satisfying

- $A_{j}$ has eigenvalues $\pm \frac{1}{4}$, i.e. $\operatorname{det}\left(A_{j}\right)=-1 / 16$,

$$
\text { - } A_{\infty}=-A_{1}-A_{2}-A_{3}=\left(\begin{array}{cc}
-\frac{1}{4} & 0 \\
0 & \frac{1}{4}
\end{array}\right) \text {. }
$$

The latter normalization is obtained under $\mathrm{SL}_{2}$-action and the categorical quotient of those systems under the remaining diagonal conjugacy is the affine threefold $P$ defined by those $\left(t, a_{1}, a_{2}, H\right) \in \mathbb{C}^{4}, H=b_{2} c_{1}$, satisfying

$$
H^{2}+2 H\left(a_{1}-1 / 4\right)\left(a_{2}-1 / 4\right)+\left(a_{1}^{2}-1 / 16\right)\left(a_{2}^{2}-1 / 16\right)=0
$$

and $t \neq 0,1$. This is our parameter space. The special family of connections on $\mathcal{O}(1) \oplus \mathcal{O}(-1)$ is computed in Section 6, and in Section 9 we compare it with the classical Okamoto moduli space.

In order to understand the monodromy representation of an irreducible Lamé connection, one observes that it can be lifted as a representation of the fundamental group of the elliptic curve $E_{t}: w^{2}=$ $z(z-1)(z-t)$, via the projection

$$
E_{t} \rightarrow \mathbb{P}^{1}:(z, w) \mapsto z,
$$


into $S L_{2}$ : the monodromy or Galois group has an index 2 abelian subgroup and is therefore either reducible or dihedral. In fact, any irreducible Lamé connection $\nabla$ can be derived by pushing forward a regular rank 1 connection $\tilde{\nabla}$ on $E_{t}$. An analytic proof of those facts was given by N.J. Hitchin in [7]. We provide in Sections 1 and 2 a more algebraic proof. Now, the monodromy representation of $\tilde{\nabla}$ may be viewed as an element of $\mathbb{C}^{*} \times \mathbb{C}^{*}$, once generators for $\pi_{1}\left(E_{t}\right)$ are fixed. The Riemann-Hilbert correspondence for rank 1 connections on the elliptic curve $E_{t}$ is very classical and is studied in Section 3: in this case it is an analytic homomorphism of groups, where the group law for rank 1 connections is the tensor product ([22], pp. 384-385) . Now, the Galois group of $\nabla$ is finite iff the Galois group of $\tilde{\nabla}$ is. It is a remarkable fact (see Proposition 3.2) that a Lamé connection $\nabla$ has non trivial underlying vector bundle iff the corresponding rank 1 connection $\tilde{\nabla}$ on $E_{t}$ is defined on the trivial line bundle. As a consequence, the vector bundle of a Lamé connection with Galois group $D_{N}^{S L_{2}}$ is free and the locus of $D_{N}^{S L_{2}}$ is entirely contained in our parameter space $P$.

There are several ways to compute the locus for $D_{N}^{S L_{2}}$. First of all, one can compute factors of the $N^{t h}$ symetric power of $\nabla$ and look for a rational solution. This is discussed at the end of Section 1 but this approach leads to heavy computations and, despite the help of J.-C. Faugère, we were not able to go beyond $N=3$. However, this approach turns out to be useful for the computation of the minimal polynomial of the corresponding field extension.

A more efficient approach is to compute the push forward $\tilde{\nabla}$ and decide when its $N^{\text {th }}$ tensor product is trivial. Let $\tilde{\nabla}$ be given by the formula

$$
\tilde{\nabla} f=\left(c+\frac{w+w_{0}}{z-z_{0}}\right) \frac{d z}{2 w} \otimes f
$$

where $\left(z_{0}, w_{0}\right) \in E_{t}$ is the point defining the underlying line bundle and $c \in \mathbb{C}$ a constant. A necessary condition is that the $N^{t h}$ tensor product of the line bundle is itself trivial, i.e., $\left(z_{0}, w_{0}\right)$ is an $N$-order point of $E_{t}$. Next it remains to find the unique value of $c$ for which there exists a rational function $f$ such that $\frac{d f}{f}=N\left(c+\frac{w+w_{0}}{z-z_{0}}\right) \frac{d z}{2 w}$. In Section 7 , we give simple formulae which permit to express those conditions in the initial parameters $\left(a_{1}, a_{2}, H\right)$ of Lamé systems. However, this determination of $c$ still leads to tedious computations as $N$ increases.

In [6], N.J. Hitchin uses this later approach to construct algebraic solutions of the Painlevé VI equation. Inspired by [13], we use the other direction here. Using a special solution of Painlevé VI, we obtain that if $\left(z_{0}(t), w_{0}(t)\right)$ is a point of finite order on $E_{t}$, with varying $t$, then the 
differential Galois group is finite if and only if

$$
c=\frac{z_{0}\left(z_{0}-1\right)-t(t-1) \frac{d z_{0}}{d t}}{w_{0}} .
$$

We use the above ideas to provide explicit parametrizations for $D_{2}^{S L_{2}}$, $D_{3}^{S L_{2}}$ and $D_{4}^{S L_{2}}$. For instance, $D_{2}^{S L_{2}}$ is given by points of order 4 on $E_{t}$, that is those points $\left(z_{0}, w_{0}\right)$ of $E_{t}$ satisfying

$$
\left(z_{0}^{2}-t\right)\left(z_{0}^{2}-2 z_{0}+t\right)\left(z_{0}^{2}-2 t z_{0}+t\right)=0 .
$$

For each $t \neq 0,1, \infty$ we will find 6 systems with Galois group $D_{2}^{S L_{2}}$, but there are in fact 3 irreducible families of them for varying $t$. The one corresponding to the first factor can be parametrized by $\lambda=\sqrt{t}$ and the resulting Lamé connection is for instance given by

$$
\frac{d}{d z}+\left(\begin{array}{cc}
\frac{(z-\lambda)\left(2 z-\lambda^{2}-1\right)}{8 z(z-1)\left(z-\lambda^{2}\right)} & \frac{\left(1-\lambda^{2}\right)(z+\lambda)}{8 z(z-1)\left(z-\lambda^{2}\right)} \\
\frac{\left(1-\lambda^{2}\right)(3 z-\lambda)}{8 z(z-1)\left(z-\lambda^{2}\right)} & -\frac{(z-\lambda)\left(2 z-\lambda^{2}-1\right)}{8 z(z-1)\left(z-\lambda^{2}\right)}
\end{array}\right)
$$

In our moduli space $P_{t}$, this family is parametrized by

$$
t=\lambda^{2}, \quad a_{1}=\frac{\lambda^{2}+1}{8 \lambda}, \quad a_{2}=\frac{1-\lambda}{8} \quad \text { and } \quad H=\frac{(\lambda+1)\left(\lambda^{2}-1\right)}{64 \lambda}
$$

or equivalently defined by the equations

$1+32 a_{1} a_{2}-4 a_{1}+32 a_{2}^{2}-8 a_{2}=0$ and $32 H+4 a_{1}-32 a_{2}^{2}+16 a_{2}-1=0$.

Our approach also allows to find the irreducible polynomials for the corresponding Picard-Vessiot extension:

$$
Y^{8}-2 \frac{k_{1}\left(-z^{2}+\left(2-2 \lambda+2 \lambda^{2}\right) z-\lambda^{2}\right) Y^{4}}{(z-1) z\left(-z+\lambda^{2}\right)}+\frac{k_{1}(z-\lambda)^{4}}{z^{2}(z-1)^{2}\left(z-\lambda^{2}\right)^{2}}
$$

producing a polynomial over $\mathbb{C}(z)$, depending on two parameters, whose galois group is the quaternion group $D_{2}^{S L_{2}}$ of order 8 for all non confluent parameter values.

For all $n \in \mathbb{Z}$, the classical Lamé equation

$$
\mathcal{L}_{n, B}: \frac{d^{2} y}{d z^{2}}+\frac{1}{2} \frac{f^{\prime}}{f} \frac{d y}{d z}-\frac{n(n+1) z+B}{f} y, \quad B \in \mathbb{C}
$$

with $f=4\left(z-e_{1}\right)\left(z-e_{2}\right)\left(z-e_{3}\right)$ and $e_{1}+e_{2}+e_{3}=0$ is gauge equivalent to a two parameter subfamily of $P$ where $t=\frac{e_{3}-e_{1}}{e_{2}-e_{1}}$. Therefore, once we determine the locus of those Lamé connections having Galois group $D_{N}^{S L_{2}}$, we deduce by restriction all classical Lamé equations having this Galois group. This provides a new approach for a very classical subject (see [4], [5] and references therein). For instance, the subfamily of Lamé systems defined by $\mathcal{L}_{2, B}$ intersects twice the irreducible 
component of $D_{2}^{S L_{2}}$ presented above, namely for $t=-1$ and $b^{2}=\frac{72}{7}$, where $B=\left(e_{2}-e_{1}\right) b$.

\section{IMPRIMITIVE DIFFERENTIAL MODULES OF RANK 2}

Let $K$ denote a differential field. The field of constants $C$ of $K$ is supposed to have characteristic 0 and to be different from $K$. Let $M$ be a differential module over $K$ of dimension 2 and let $B_{1}, B_{2}$ be a basis of $M$ over $K$. Any element $m_{1} \otimes m_{2}$ of the second symmetric power sym $m_{K}^{2} M$ will be written as $m_{1} m_{2}$. In particular $B_{1}^{2}, B_{1} B_{2}, B_{2}^{2}$ is a basis of sym $^{2} M$. Let $L \supset K$ be an extension of degree 2 and let $N$ denote a 1-dimensional differential module over $L$. We write $\operatorname{Res}_{L / K}(N)$ for $N$ viewed as 2-dimensional differential module over $K$.

Lemma 1.1. Let $F=\alpha_{1} B_{1}^{2}+\alpha_{2} B_{1} B_{2}+\alpha_{3} B_{2}^{2} \neq 0$ generate a differential submodule of sym ${ }_{K}^{2} M$. One regards $F$ as element of the polynomial ring $K\left[B_{1}, B_{2}\right]$. Put $\Delta=\alpha_{2}^{2}-4 \alpha_{1} \alpha_{3}$. Then:

(1) If $F$ is a $K^{*}$-multiple of a square (or equivalently $\Delta=0$ ), then we may suppose that $F=B_{1}^{2}$. Now $K B_{1}$ is a differential submodule of $M$.

(2) If $F$ is a product of two distinct linear factors (or equivalently $\Delta \neq 0$ is a square in $K$ ), then we may suppose that $F=B_{1} B_{2}$. Now $K B_{1}$ and $K B_{2}$ are differential submodules of $M$.

(3) Suppose that $F$ is irreducible. We may assume $F=B_{2}^{2}-\Delta B_{1}^{2}$. Put $L=K(\delta)$ with $\delta^{2}=\Delta$. There exists a 1-dimensional module $N=$ Le over $L$ with $M \cong \operatorname{Res}_{L / K}(N)$ and such that the kernel of the surjective (obvious) morphism of differential modules sym ${ }_{K}^{2} M \rightarrow$ $\operatorname{Res}_{L / K}\left(\operatorname{sym}_{L}^{2} N\right)$ equals $K F$.

The differential module $N=$ Le with $\partial e=u e$, with the above properties, is unique up to conjugation, i.e., $u \in L$ is replaced by its conjugate $\bar{u}$.

Moreover sym ${ }_{K}^{2} M$ is the direct sum of $K\left(B_{2}^{2}-\Delta B_{1}^{2}\right)$ and the differential submodule $K\left(B_{2}^{2}+\Delta B_{1}^{2}\right)+K\left(B_{1} B_{2}\right)$.

(4) Suppose that $C$ is algebraically closed and that $M$ is irreducible and (for convenience) that the determinant of $M$ (i.e., the exterior product $\left.\Lambda^{2} M\right)$ is trivial. Then sym $_{K}^{2} M$ contains more than one 1-dimensional submodule, if and only if the differential Galois group of $M$ is $D_{2}^{\mathrm{SL}_{2}}$. Moreover, in this case there are precisely three 1-dimensional submodules of sym $_{K}^{2} M$ and each one defines a different quadratic extension of $K$.

Proof. (1) and (2) follow by straightforward computation. In case (3) we give $M$ the structure of a vector space over $L$ by prescribing $\delta B_{1}=$ $B_{2}$ and $\delta B_{2}=\Delta B_{1}$. Thus we obtain a vector space $N=L B_{1}$ over 
$L$. Let $\partial$ on $M$ satisfy $\partial B_{1}=a B_{1}+b B_{2}$ with $a, b \in K$. Then $\partial$ on $N$ is defined by $\partial B_{1}=(a+b \delta) B_{1}$. From the assumption that $\partial\left(B_{2}^{2}-\right.$ $\left.\Delta B_{1}^{2}\right)$ is a multiple of $\left(B_{2}^{2}-\Delta B_{1}^{2}\right)$, it follows that $\operatorname{Res}_{L / K}$ is isomorphic to $M$. The obvious morphism $\operatorname{sym}_{K}^{2} M \rightarrow \operatorname{Res}_{L / K}(N)$ is given by $B_{1}^{2}, B_{1} B_{2}, B_{2}^{2}$ have images $B_{1}^{2}, \delta B_{1}^{2}, \delta^{2} B_{1}^{2}$. The map is surjective and its kernel is generated by $B_{2}^{2}-\Delta B_{1}^{2}$. The only freedom one has in giving $M$ a structure of differential module over $L$ is by prescribing $\partial B_{1}=(a-b \delta) B_{1}$. The rest of the statement (3) follows by easy computation.

The first statement of (4) is well known and follows in fact from the classification of the algebraic subgroups of $\mathrm{Sl}_{2}(C)$. Suppose that the differential Galois group of $M$ is $D_{2}^{\mathrm{Sl}_{2}}$. Then the differential Galois group of $s y m^{2} M$ is the group $D_{2}$ (of order 4) and $s y m^{2} M$ is the direct sum $L_{1} \oplus L_{2} \oplus L_{3}$ of submodules. Each $L_{j}$ corresponds to a different surjective homomorphism $\chi_{j}: D_{2} \rightarrow\{ \pm 1\}$. The quadratic extension of $K$ corresponding to $L_{j}$ is the fixed field of the Picard-Vessiot field under the kernel of $\chi_{j}$.

In case (3) of Lemma 1.1, the differential module will be called imprimitive.

Remark. In case (4) of Lemma 1.1, the condition that $C$ is algebraically closed cannot be omitted.

Corollary 1.2. Let $M$ be an irreducible differential module of dimension 2 over $K$. Then $M$ is imprimitive if and only if there exists a quadratic extension $L \supset K$ such that $L \otimes_{K} M$ has a 1-dimensional submodule.

Proof. Suppose that $M$ is imprimitive. Then $M=\operatorname{Res}_{L / K}(N)$ for suitable $L$ and $N$. The kernel of the canonical map $L \otimes_{K} \operatorname{Res}_{L / K}(N) \rightarrow$ $L \otimes_{L} N=N$ is a 1-dimensional submodule of $L \otimes_{K} M$.

On the other hand, suppose that $L \otimes_{K} M$ has a 1-dimensional submodule $T_{1}$. Using the conjugation of $L / K$ one finds another 1-dimensional submodule $T_{2}$. The 1-dimensional submodule $T_{1} \otimes_{L} T_{2}$ of $L \otimes_{K} s y m_{K}^{2} M$ is invariant under the conjugation of $L / K$ and thus provides a 1dimensional submodule of $s^{2} M$.

Suppose that $M$ is an imprimitive differential module over $K$ of dimension 2. For every integer $n \geq 2$, the differential module $s^{\prime} m_{K}^{n} M$ has an explicit 2-dimensional factor (direct summand) $\operatorname{Res}_{L / K}\left(\operatorname{sym}_{L}^{n} N\right)$. We use the notation of Lemma 1.1, i.e., $L=K(\delta), \delta^{2}=\Delta, N=L B_{1}$ and $\partial B_{1}=(a+b \delta) B_{1}$. Then $\operatorname{sym}_{L}^{n} N$ has generator $e=B_{1}^{n}$ and 
$\partial e=n(a+b \delta) e$. Then $\operatorname{Res}_{L / K}\left(\operatorname{sym}_{L}^{n} N\right)$ has basis $e, \delta e$ and the matrix of $\partial$ with respect to this basis reads

$$
\left(\begin{array}{cc}
n a & n b \Delta \\
n b & n a+\frac{\Delta^{\prime}}{2 \Delta}
\end{array}\right)
$$

The above method to find a factor of the $n$th symmetric power can also be given explicitly for scalar equations. Consider a scalar equation $y^{\prime \prime}+a_{1} y^{\prime}+a_{0} y=0$ over $K$ with $C$ algebraically closed, such that its differential Galois is an irreducible subgroup of $D_{\infty}^{\mathrm{SL}_{2}}$. The (abstract) solution space has a basis $y_{1}, y_{2}$ such that the differential Galois group permutes the two lines $C y_{1}, C y_{2}$ (cf. Lemma 1.1 (3)). Then $S=y_{1} y_{2}$ is a solution of the second symmetric power and $S^{2} \in K$. This second symmetric power reads

$$
y^{\prime \prime \prime}+3 a_{1} y^{\prime \prime}+\left(4 a_{0}+2 a_{1}^{2}+a_{1}^{\prime}\right) y^{\prime}+\left(2 a_{0}^{\prime}+4 a_{0} a_{1}\right) y=0 .
$$

The term $\frac{S^{\prime}}{S}$ can be computed explicitly using Kovacic's algorithm. As a consequence the expression $\frac{S^{\prime \prime}}{S}=\left(\frac{S^{\prime}}{S}\right)^{\prime}+\left(\frac{S^{\prime}}{S}\right)^{2}$ is known as element of $K$. Further $\frac{y_{1}^{\prime}}{y_{1}}$ and $\frac{y_{2}^{\prime}}{y_{2}}$ satisfy the polynomial equation

$$
U^{2}-\frac{S^{\prime}}{S} U+\left(a_{0}+a_{1} \frac{S^{\prime}}{2 S}+\frac{S^{\prime \prime}}{2 S}\right)=0 \text { over } K .
$$

From this, using elimination, one derives a differential equation $L_{n}$ for $y_{1}^{n}$ and $y_{2}^{n}$.

$L_{n}(y)=y^{\prime \prime}+\left(a_{1}+(1-n) \frac{S^{\prime}}{S}\right) y^{\prime}+\left(n^{2} a_{0}+\left(n^{2}-n\right) a_{1} \frac{S^{\prime}}{2 S}+\left(n^{2}-n\right) \frac{S^{\prime \prime}}{2 S}\right) y$.

The differential Galois group is finite if and only if $L_{n}(y)=0$ has a rational solution for some integer $n>1$. In this case the group has two polynomial invariants, one given by $\left(y_{1} y_{2}\right)^{2}$ and one given by $y_{1}^{2 n}+(-1)^{n} y_{2}^{2 n}$ whose value is a rational solution of $L_{n}(y)=0$. Since the stabiliser of a vector under a finite subgroup of $\mathrm{SL}_{2}$ must be trivial, the length of the orbit of a vector is always the group order. Therefore the minimal polynomial of $y_{1}$ must be

$$
\left.\prod_{\sigma \in G}\left(Y-\sigma\left(y_{1}\right)\right)\right)=Y^{4 n}-\left(y_{1}^{2 n}+(-1)^{n} y_{2}^{2 n}\right) Y^{2 n}+(-1)^{n}\left(\left(y_{1} y_{2}\right)^{2}\right)^{n}
$$

Once a connection with finite differential Galois group is known, the computation of a rational solution $g \in \mathbb{C}(z)$ of the second order equation $L_{n}(y)=0$ gives a very efficient way to compute the minimal polynomial of a primitive element of the corresponding field extension. The value of $y_{1}^{2 n}+(-1)^{n} y_{2}^{2 n}$ and of $g$ may differ by a constant that can be determined using series computations at a regular point. 


\section{Connections with 4 Special Singular points}

Consider an irreducible connection of rank 2 on $\mathbb{P}_{C}^{1}$ having 4 singular points $0,1, t, \infty$ and local exponents $1 / 4,-1 / 4$ for each singular point. The generic fibre of this connection is a differential module $M$ of dimension 2 over $C(z)$, the field of rational functions of $\mathbb{P}_{C}^{1}$.

Proposition 2.1. The module $M$ is imprimitive and there is a 1dimensional submodule of $\operatorname{sym}^{2} M$ such that the corresponding quadratic extension $L \supset C(z)$ has the form $C(z)(w)$ with $w^{2}=c z(z-$ 1) $(z-t)$ and $c \in C^{*}$.

A direct proof by computation will be given later on. First we consider the case where $C$ is the field of complex numbers $\mathbb{C}$ and we present an analytic proof. We start by investigating the monodromy of $M$. One chooses a base point in $\mathbb{P}_{\mathbb{C}}^{1} \backslash\{0,1, t, \infty\}$ and generators $\lambda_{1}, \ldots, \lambda_{4}$ of the fundamental group $\pi_{1}$ of this space, consisting of loops around the points $0,1, t, \infty$ such that $\lambda_{1} \cdots \lambda_{4}=1$. The monodromy is a homomorphism $\pi_{1} \rightarrow \mathrm{Sl}_{2}(\mathbb{C})$, sending each $\lambda_{j}$ to an element $B_{j}$ of order 4 . The monodromy is therefore described by elements $B_{1}, \ldots, B_{4} \in \mathrm{Sl}_{2}(\mathbb{C})$ of order 4 with $B_{1} \cdots B_{4}=1$.

Lemma 2.2. Let $B_{1}, \ldots, B_{4} \in \mathrm{Sl}_{2}(\mathbb{C})$ satisfy $B_{1} \cdots B_{4}=1$ and $B_{j}^{4}=1$ for every $j$. Let $G$ be the group generated by the $B_{j}$. There are two possibilities:

(i) $G$ is reducible and contained in a Borel subgroup of $\mathrm{Sl}_{2}(\mathbb{C})$.

(ii) $G$ is irreducible and contained in $D_{\infty}^{\mathrm{Sl}_{2}}$.

Proof. In an appropriated basis the matrix $B_{i}$ is diagonal and thus $\left(B_{i}\right)^{2}= \pm 1$. Therefore $\left(B_{i}\right)^{2}$ is in the center of $\mathrm{Sl}_{2}(\mathbb{C})$. From $\varphi: \mathrm{Sl}_{2}(\mathbb{C}) \rightarrow$ $\mathrm{PSl}_{2}(\mathbb{C})$ we get that $\varphi(G)$ is a group generated by the elements $\varphi\left(B_{j}\right)$ with the property that $\varphi\left(B_{j}\right)^{2}=1$ and $\varphi\left(B_{1}\right) \cdots \varphi\left(B_{4}\right)=1$. From [4] proof of Theorem 3.3 we get that $G$ is projectively equivalent to a Dihedral group or a Borel group, showing the result.

Second proof. Let $E$ denote the elliptic curve given by the affine equation $w^{2}=z(z-1)(z-t)$. Let $p_{1}, \ldots, p_{4} \in E$ denote the points of $E$ with images $0,1, t, \infty$. Then $E_{-}:=E \backslash\left\{p_{1}, \ldots, p_{4}\right\} \rightarrow \mathbb{P}_{-}^{1}:=\mathbb{P}^{1} \backslash\{0,1, t, \infty\}$ is a covering of degree 2 and the homomorphism $I: \pi_{1}\left(E_{-}\right) \rightarrow \pi_{1}\left(\mathbb{P}_{-}^{1}\right)$ is injective and its image is a subgroup of index 2. Write, as before, $\pi_{1}\left(\mathbb{P}_{-}^{1}\right)=<\lambda_{1}, \ldots, \lambda_{4} \mid \lambda_{1} \cdots \lambda_{4}=1>$. Then the image of $I$ is the kernel of the homomorphism $\pi_{1}\left(\mathbb{P}_{-}^{1}\right) \rightarrow\{ \pm 1\}$ that sends each $\lambda_{j}$ to -1 . One can give $\pi_{1}\left(E_{-}\right)$generators $a, b, \mu_{1}, \ldots, \mu_{4}$ such that:

(i) Each $\mu_{j}$ is a loop around $p_{j}$ and $I$ maps $\mu_{j}$ to a conjugate of $\lambda_{j}^{2}$.

(ii) There is only one relation, namely $\mu_{1} \mu_{2} \mu_{3} \mu_{4} a b a^{-1} b^{-1}=1$. 
(iii) The images of $a, b$ in $\pi_{1}(E)$ under the canonical map $\pi_{1}\left(E_{-}\right) \rightarrow$ $\pi_{1}(E)$ are generators of $\pi_{1}(E)$.

(iv) Conjugation with an (or any) element $\alpha \in \pi_{1}\left(\mathbb{P}_{-}^{1}\right)$, not contained in $\pi_{1}\left(E_{-}\right)$, acts as -1 on the abelianized group $\left(\pi_{1}\left(E_{-}\right)\right)_{a b}$.

We use the map $I$ to identify $\pi_{1}\left(E_{-}\right)$with a subgroup of $\pi_{1}\left(\mathbb{P}_{-}^{1}\right)$.

Consider a homomorphism $h: \pi_{1}\left(\mathbb{P}_{-}^{1}\right) \rightarrow \mathrm{SL}_{2}(\mathbb{C})$ which sends each $\lambda_{j}$ to $B_{j}$. Then $h\left(\mu_{j}\right)=-1$ for all $j$ and $h(a)$ commutes with $h(b)$. In particular, $h\left(\pi_{1}\left(E_{-}\right)\right)$is an abelian subgroup of $\mathrm{SL}_{2}(\mathbb{C})$. Further, $h$ induces a homomorphism $\pi_{1}(E) \rightarrow \mathrm{SL}_{2}(\mathbb{C})$ which will also be called $h$. There are the following possibilities:

(a) This abelian subgroup has precisely one invariant line $\mathbb{C} e$ in $\mathbf{C}^{2}$. Then $\mathbb{C} e$ is also invariant under $h\left(\pi_{1}\left(\mathbb{P}_{-}^{1}\right)\right)$ and the representation $h$ is reducible.

(b) This abelian subgroup has precisely two invariant lines $\mathbb{C} e_{1}, \mathbf{C} e_{2}$. Then $h: \pi_{1}(E) \rightarrow \mathrm{SL}_{2}(\mathbb{C})$ has the form $h(c) e_{1}=\chi(c) e_{1}$ and $h(c) e_{2}=$ $\chi(c)^{-1} e_{2}$, for some character $\chi$ of $\pi_{1}(E) \cong \mathbb{Z}^{2}$ such that $\chi^{2} \neq 1$. For any $\alpha \in \pi_{1}\left(\mathbb{P}_{-}^{1}\right)$, not contained in $\pi_{1}\left(E_{-}\right), h(\alpha)$ interchanges the two lines $\mathbb{C} e_{1}$ and $\mathbb{C}_{2}$. This follows from (iv). In particular, the representation $h$ is irreducible and its image is an irreducible subgroup of $D_{\infty}^{\mathrm{Sl}_{2}}$.

(c) This abelian subgroup has more than two invariant lines. Then $h$ maps every element of $\pi_{1}\left(E_{-}\right)$to \pm 1 . Then $h\left(\pi_{1}\left(\mathbb{P}_{-}^{1}\right)\right)$ is abelian (actually cyclic of order 4) and the representation is reducible.

Observations 2.3. We supplement the information of Lemma 2.2 with a well known description of the monodromy. The monodromy tuple $\left(B_{1}, \ldots, B_{4}\right)$, associated to a differential module as above, is only determined up to simultaneous conjugation. The collection $\mathcal{T}$ of all tuples $\left(B_{1}, \ldots, B_{4}\right) \in \mathrm{Sl}_{2}^{4}$ satisfying $B_{j}^{4}=1$ for every $j$ and $B_{1} \cdots B_{4}=1$ is a closed subset of $\mathrm{Sl}_{2}^{4}$. The group $\mathrm{PSL}_{2}$ acts on $\mathcal{T}$ by conjugation. There is a categorical quotient, namely $\mathcal{Q}=\operatorname{Spec}\left[t_{1}, t_{2}, t_{3}\right] /\left(t_{1}^{2}+t_{2}^{2}+\right.$ $\left.t_{3}^{2}+t_{1} t_{2} t_{3}-4\right)$, where the relation is a special case of the Fricke Klein formula. The affine cubic surface can be identified with the classical Cayley cubic surface. The morphism $q: \mathcal{T} \rightarrow \mathcal{Q}$ is given by

$$
\left(B_{1}, \ldots, B_{4}\right) \mapsto\left(t_{1}=\operatorname{tr}\left(B_{1} B_{2}\right), t_{2}=\operatorname{tr}\left(B_{2} B_{3}\right), t_{3}=\operatorname{tr}\left(B_{1} B_{3}\right)\right) .
$$

$S=\{(-2,2,2),(2,-2,2),(2,2,-2),(-2,-2,-2)\}$ is the set of the singular points of $\mathcal{Q}$. The preimage of $S$ is the locus in $\mathcal{T}$ describing the reducible groups (this follows easily from the proof of Lemma 2.2). Let $\mathcal{T}_{\text {irred }}$ denote the open subset corresponding to the irreducible groups. Then $q: \mathcal{T}_{\text {irred }} \rightarrow \mathcal{Q} \backslash S$ is a geometric quotient for the action of the group $\mathrm{PSL}_{2}$ (this follows easily from the second proof of Lemma 2.2). 
In particular, $\mathcal{T}_{\text {irred }}$ is reduced, irreducible, smooth and has dimension 5 .

The second proof of Lemma 2.2 leads to a connection between the monodromy of the elliptic curve $E$ and the special monodromy for $\mathbb{P}_{-}^{1}$. Let a homomorphism $\chi: \pi_{1}(E) \rightarrow \mathbb{C}^{*}$, with $\chi^{2} \neq 1$ be given. Define $h: \pi_{1}\left(E_{-}\right) \rightarrow \mathrm{SL}_{2}(\mathbb{C})$ by $h\left(\mu_{j}\right)=-1$ for every $j$ and $h(x)$ is the diagonal matrix with entries $\chi(x), \chi(x)^{-1}$, for $x=a, b$. This homomorphism can be extended to a homomorphism $\phi: \pi_{1}\left(\mathbb{P}_{-}^{1}\right) \rightarrow$ $\mathrm{SL}_{2}(\mathbb{C})$ as follows: for any $x \in \pi_{1}\left(E_{-}\right)$one defines $\psi(x)=h(x)$ (of course) and $\psi\left(x \lambda_{4}\right)=h(x) \cdot\left(\begin{array}{cc}0 & 1 \\ -1 & 0\end{array}\right)$. Using (iv), one easily verifies that $\psi$ is indeed a homomorphism.

We note that two characters $\chi_{1}, \chi_{2}: \pi_{1}(E) \rightarrow \mathbb{C}^{*}$ with $\chi_{1}^{2} \neq 1 \neq \chi_{2}^{2}$ induce isomorphic irreducible representations $\rho_{1}, \rho_{2}: \pi_{1}\left(\mathbb{P}_{-}^{1}\right) \rightarrow \mathrm{Sl}_{2}(\mathbb{C})$ if and only if $\chi_{2} \in\left\{\chi_{1}, \chi_{1}^{-1}\right\}$. We see $T_{E}:=\operatorname{Hom}\left(\pi_{1}(E), \mathbb{C}^{*}\right)$ as an algebraic torus with group of characters $\pi_{1}(E)$. Let $T_{E}[2]$ denote the subgroup of the points of order dividing 2. Consider $\left(T_{E} \backslash T_{E}[2]\right) / \sim$, where $\chi_{1} \sim \chi_{2}$ if and only if $\chi_{2} \in\left\{\chi_{1}, \chi_{1}^{-1}\right\}$. The above construction induces an isomorphism of algebraic varieties

$$
\left(T_{E} \backslash T_{E}[2]\right) / \sim \longrightarrow \mathcal{Q} \backslash S .
$$

This isomorphism extends to an isomorphism $T_{E} / \sim \longrightarrow \mathcal{Q}$.

The isomorphism can be made explicit as follows. One considers an element $\left(B_{1}, \ldots, B_{4}\right) \in \mathcal{T}$. Suppose that this tuple generates an irreducible subgroup, then the tuple is an example for case (b) in the second proof of Lemma 2.2. In the conjugacy class of the tuple there is an element of the form

$$
\left(\left(\begin{array}{cc}
0 & r \\
-1 / r & 0
\end{array}\right),\left(\begin{array}{cc}
0 & s \\
-1 / s & 0
\end{array}\right),\left(\begin{array}{cc}
0 & t \\
-1 / t & 0
\end{array}\right),\left(\begin{array}{cc}
0 & 1 \\
-1 & 0
\end{array}\right)\right)
$$

with $s=r t$ because $B_{1} \cdots B_{4}=1$. Moreover, $(r, t) \neq( \pm 1, \pm 1)$. The above element is unique up to conjugation with $\left(\begin{array}{cc}0 & 1 \\ -1 & 0\end{array}\right)$. This operation changes $(r, t)$ into $(1 / r, 1 / t)$. Thus we find an isomorphism between $\left\{\left(\mathbb{C}^{*}\right)^{2} \backslash\{( \pm 1, \pm 1)\}\right\} / \sim$ and $\mathcal{Q} \backslash S$, which is essentially the same as the above isomorphism.

Proof of Proposition 2.1. We first consider the case where $C$ is the field of complex numbers $\mathbb{C}$. According to Lemma 2.2, the monodromy group of $M$, generated by $B_{1}, \ldots, B_{4}$, is an irreducible subgroup of $D_{\infty}^{\mathrm{Sl}_{2}}$ and the same holds for its Zariski closure which is the differential Galois group. It follows that $\operatorname{sym}^{2} M$ contains a 1-dimensional submodule. 
In case the differential Galois group $G$ is $D_{\infty}^{\mathrm{Sl}_{2}}$ or $D_{n}^{\mathrm{Sl}_{2}}$ with $n>2$, there is only one 1-dimensional submodule. The corresponding field extension $L \supset \mathbb{C}(z)$ corresponds to the unique surjective homomorphism $h: G \rightarrow\{ \pm 1\}$. The elements $B_{j} \in G$ have order 4 and it follows that $h\left(B_{j}\right)=-1$ for every $j$. This implies that $L \supset \mathbb{C}(z)$ is ramified above each of the points $0,1, t, \infty$. The extension $L$ is therefore given by $L=\mathbb{C}(z)(w)$ with $w^{2}=z(z-1)(z-t)$.

If the differential Galois group $G$ is $D_{2}^{\mathrm{Sl}_{2}}$, then there are precisely three 1-dimensional submodules of $s y m^{2} M$ and there are also precisely three surjective homomorphisms $h: G \rightarrow\{ \pm 1\}$. As above, it suffices to verify that there exists a homomorphism with $h\left(B_{j}\right)=-1$ for all $j$. The commutator subgroup of $G$ is identical with the center of $G$. Consider the images $b_{1}, \ldots, b_{4} \in \mathrm{PSl}_{2}$ of $B_{1}, \ldots, B_{4}$. They generate a commutative group with two generators, say $A$ and $B$, each with order two. One has $b_{j} \in\{A, B, A B\}$ for all $j$. Further the surjective homomorphisms $h: G \rightarrow\{ \pm 1\}$ are given by $(h(A), h(B))=(-1,-1),(-1,1),(1,-1)$. Up to a choice for $A$ and $B$ there are three possibilities:

(i) $\left(b_{1}, b_{2}, b_{3}, b_{4}\right) \mapsto(A, B, A, B)$. The possibilities for $L \supset \mathbb{C}(z)$ are given by the sets of ramified points $\{0,1, t, \infty\},\{0, t\},\{1, \infty\}$.

(ii) $\left(b_{1}, b_{2}, b_{3}, b_{4}\right) \mapsto(A, B, B, A)$. The possibilities for the sets of ramified points are $\{0,1, t, \infty\},\{0, \infty\},\{1, t\}$.

(iii) $\left(b_{1}, b_{2}, b_{3}, b_{4}\right) \mapsto(A, A, B, B)$. The possibilities for the sets of ramified points are $\{0,1, t, \infty\},\{0,1\},\{t, \infty\}$.

In particular, in each case one of the corresponding fields is $\mathbb{C}(z)(w)$ with $w^{2}=z(z-1)(z-t)$. In the algorithmic part of this paper we will verify that each of the cases (i)-(iii) are present in our family.

Now we consider a general case. We may suppose that the algebraic closure $\bar{C}$ of $C$ is a subfield of $\mathbb{C}$. Let $M^{+}$denote the differential module $\bar{C}(z) \otimes_{C(z)} M$. For this differential module the statement of the proposition follows easily from the statement for the case $\mathbb{C}$. Further $\operatorname{sym}^{2} M^{+}$has one or three 1-dimensional submodules. They produce one or three distinct quadratic extensions of $\bar{C}(z)$. It follows that the Galois group of $\bar{C} / C$ preserves these 1-dimensional submodules. Therefore $s^{2} m^{2} M$ has one or three 1-dimensional submodules. For one of them the field extension is over $\bar{C}(z)$ given by $w^{2}=z(z-1)(z-t)$. Thus over the field $C(z)$ this equation reads $w^{2}=c z(z-1)(z-t)$ for some $c \in C^{*}$.

\section{Regular conneCtions on an Elliptic CuRve}

In this section the base field will be $\mathbb{C}$, the field of complex numbers. Let $M$ denote a differential module over $\mathbb{C}(z)$ with four singular points 
$0,1, t, \infty$ and local exponents $1 / 4,-1 / 4$ for each of them. According to Proposition 2.1, $M$ is the restriction to $\mathbb{C}(z)$ of a differential module of rank one over the field $\mathbb{C}(z, w)$ with $w^{2}=z(z-1)(z-t)$. After tensoring $M$ with the differential module $(\mathbb{C}(z) e, \partial)$, where $\partial(e)=\left(\frac{1 / 4}{z}+\frac{1 / 4}{z-1}+\right.$ $\left.\frac{1 / 4}{z-t}\right) e$, the new differential module has local exponents $1 / 2,0$ at each singular point. The corresponding rank one differential module over $\mathbb{C}(z, w)=\mathbb{C}(E)$ has no singularities. Here $E$ denotes the elliptic curve given by the equation $w^{2}=z(z-1)(z-t)$. We start by describing the regular connections on $E$.

A regular connection is a pair $(\mathcal{L}, \nabla)$ of a line bundle on $E$ and a connection $\nabla: \mathcal{L} \rightarrow \Omega \otimes \mathcal{L}$, where $\Omega$ denotes the sheaf of the holomorphic differentials. Its generic stalk is a vector space $\mathbb{C}(E) e$ together with a connection $\nabla$ given by $\nabla e=\omega \otimes e$ where $\omega$ of a meromorphic differential on $E$. The condition that the connection has no singularities translates into $\omega$ has only simple poles and all its residues are in $\mathbb{Z}$. Further $\omega$ is unique up to the addition of a term $\frac{d f}{f}$, with $f \in \mathbb{C}(E)^{*}$. For any meromorphic differential form $\omega$ with at most simple poles, we define $\operatorname{Res}(\omega):=\sum_{p \in E} \operatorname{res}_{p}(\omega)[p]$. This is a divisor of degree 0 on $E$ if all the residues of $\omega$ are integers. Let $\omega$ define a regular connection and let the line bundle $\mathcal{L}$ correspond to $\operatorname{Res}(\omega)$. Then $\omega$ corresponds to a global regular connection $\nabla: \mathcal{L} \rightarrow \Omega \otimes \mathcal{L}$ on $E$.

If $\mathcal{L}$ is trivial (i.e., isomorphic to $O_{E}$ ), then $\omega$ has the form $c \frac{d z}{2 w}$ for some $c \in \mathbb{C}$.

If $\mathcal{L}$ is not trivial then this line bundle corresponds to a divisor $[q]-\left[1_{E}\right]$, where $1_{E}$ is the neutral element of $E$ (as usual taken to be the point $z=\infty)$ and $q=\left(z_{0}, w_{0}\right)$ is some point $\left(\neq 1_{E}\right)$ on the affine curve given by $w^{2}=z(z-1)(z-t)$. In this case $\omega$ can be written in normalized form as $\left(c+\frac{w+w_{0}}{z-z_{0}}\right) \frac{d z}{2 w}$ with $c \in \mathbb{C}$.

Now we make a complex analytic study of the regular connections on $E$. Consider the exact sequence of sheaves on $E$

$$
0 \rightarrow \mathbb{C}^{*} \rightarrow O_{E}^{*} \rightarrow \Omega \rightarrow 0
$$

where $\mathbb{C}^{*}$ is the constant sheaf, $O_{E}^{*}$ is the sheaf of invertible holomorphic functions and $\Omega$ is the sheaf of holomorphic differential forms. The morphism $O_{E}^{*} \rightarrow \Omega$ is given by $f \mapsto \frac{d f}{f}$. This induces an exact sequence for cohomology groups

$$
0 \rightarrow \mathbb{C} \frac{d z}{2 w} \rightarrow H^{1}\left(E, \mathbb{C}^{*}\right) \rightarrow H^{1}\left(E, O_{E}^{*}\right) \rightarrow H^{1}(E, \Omega) \cdots
$$


$H^{1}\left(E, O_{E}^{*}\right)$ is the group of the equivalence classes of line bundles $\operatorname{Pic}(E)$. Its subgroup $\operatorname{Pic}^{0}(E)$ of equivalence classes of line bundles of degree 0 is identified with $E$. Let $\pi_{1}(E)$ denotes the fundamental group of $E$, then $H^{1}\left(E, \mathbb{C}^{*}\right)$ is equal to $\operatorname{Hom}\left(\pi_{1}(E), \mathbb{C}^{*}\right)$. One easily derives from the above that the following sequence is exact

$$
0 \rightarrow \mathbb{C} \frac{d z}{2 w} \rightarrow \operatorname{Hom}\left(\pi_{1}(E), \mathbb{C}^{*}\right) \rightarrow E \rightarrow 0 .
$$

Lemma 3.1. There is a natural isomorphism of groups

$$
\operatorname{Hom}\left(\pi_{1}(E), \mathbb{C}^{*}\right) \rightarrow\{(\mathcal{L}, \nabla)\} / \sim
$$

where the last group is the group of the equivalence classes of the regular connections on $E$ of rank 1.

Proof. Let $U: \mathbb{C}_{u} \rightarrow E$ denote the uniformization of $E$. Here $u$ denotes the global parameter of $\mathbb{C}$. The kernel $\Lambda$ of $U$ is identified with $\pi_{1}(E)$. Suppose that a homomorphism $\rho: \Lambda \rightarrow \mathbb{C}^{*}$ is given. Let $\mathbb{C}_{u} \times \mathbb{C}_{v}$ denote the trivial (geometric) line bundle on $\mathbb{C}_{u}$ provided with the trivial connection. Here $v$ denotes the global parameter on the linear space $\mathbb{C}$. Let $\Lambda$ act on $\mathbb{C}_{u} \times \mathbb{C}_{v}$ by $\lambda(u, v)=(u+\lambda, \rho(\lambda) v)$. The quotient by the action of $\Lambda$ is a (geometric) line bundle $\left(\mathbb{C}_{u} \times \mathbb{C}_{v}\right) / \Lambda \rightarrow \mathbb{C}_{u} / \Lambda=$ $E$ with induced connection. The sheaf of sections of this geometric line bundle is a line bundle $\mathcal{L}$ on $E$ (of degree 0 ) and is provided with a regular connection.

Let, on the other hand, a regular connection $(\mathcal{L}, \nabla)$ on $E$ be given. Then $U^{*}(\mathcal{L}, \nabla)$ is a trivial connection on $\mathbb{C}_{u}$. The group $\Lambda$ acts on the 1 dimensional solution space of this trivial connection and this produces a homomorphism $\rho: \Lambda \rightarrow \mathbb{C}^{*}$.

A slightly different way to find the isomorphism of Lemma 3.1 is the following. Consider the exact sequence of sheaves on $E$ :

$$
0 \rightarrow \mathbb{C}^{*} \rightarrow \mathcal{M}^{*} \rightarrow \Omega_{s p} \rightarrow 0,
$$

where $\mathcal{M}^{*}$ denotes the sheaf of the invertible meromorphic functions on $E$ and $\Omega_{s p}$ denotes the sheaf of the special meromorphic differentials having at most simple poles and having residues in $\mathbb{Z}$. The morphism $\mathcal{M}^{*} \rightarrow \Omega_{s p}$ is given by $f \mapsto \frac{d f}{f}$. The cohomology sequence reads

$$
0 \rightarrow \mathbb{C}^{*} \rightarrow \mathbb{C}(E)^{*} \rightarrow H^{0}\left(E, \Omega_{s p}\right) \rightarrow H^{1}\left(E, \mathbb{C}^{*}\right) \rightarrow 0 .
$$

Indeed, $H^{1}\left(E, \mathcal{M}^{*}\right)=0$ is known. The cokernel of $\mathbb{C}(E)^{*} \rightarrow H^{0}\left(E, \Omega_{s p}\right)$ has already been identified with the isomorphism classes of the regular connections on $E$. 
Proposition 3.2. Let $\pi: E \rightarrow \mathbb{P}^{1}$ denote the morphism, induced by the map $(z, w) \mapsto z$. Let $(\mathcal{L}, \nabla)$ denote a regular connection (of rank one) on $E$. Then $\pi_{*}(\mathcal{L}, \nabla)$ is a rank 2 connection on $\mathbb{P}^{1}$ having 4 regular singular points, namely $0,1, t, \infty$. At each singular point the local exponents are $0,1 / 2$. Further $\pi_{*} O_{E}$ is isomorphic to the vector bundle $O(0) \oplus O(-2)$ on $\mathbb{P}^{1}$ and $\pi_{*} \mathcal{L}$, with $\mathcal{L} \neq O_{E}$, is isomorphic to the vector bundle $O(-1) \oplus O(-1)$ on $\mathbb{P}^{1}$.

Proof. Let $U: \mathbb{C}_{u} \rightarrow E$ denote again the universal covering. Let $\tilde{\Lambda}$ denote the group of the automorphisms of $\mathbb{C}_{u}$ generated by the translations over the elements of $\Lambda$ and the map $u \mapsto-u$. Then $\mathbb{C}_{u} / \tilde{\Lambda}$ is identified with $\mathbb{P}^{1}$. The trivial connection on $\mathbb{C}_{u}$ (provided with a homomorphism $\rho: \Lambda \rightarrow \mathbb{C}^{*}$ ) yields a connection on $\mathbb{P}^{1}$ which is clearly regular outside the ramification points. For a point of ramification, say the image in $\mathbb{P}^{1}$ of the neutral element of $E$, one easily verifies that the local exponents are $0,1 / 2$. Indeed, one knows that the local exponents at a ramification point give, multiplied with the ramification index (in this case 2 ), the local exponents above. The latter are $\{0,1\}$.

A line bundle $\mathcal{L}$ on $E$ and its direct image $\pi_{*} \mathcal{L}$ have cohomology groups of the same dimension. This proves the second statement.

Remarks 3.3. From rank one connections on E to Lamé connections. Now $\pi_{*}(\mathcal{L}, \nabla)$ is tensorized with the rank one connection $T:=(T, \nabla)$ on $\mathbb{P}^{1}$ with singularities in $0,1, t, \infty$, given by $T=O([\infty]) e$ (i.e., the sheaf of meromorphic functions having at most a simple pole at $\infty$ ) and

$$
\nabla e=\left(\frac{-1 / 4}{z}+\frac{-1 / 4}{z-1}+\frac{-1 / 4}{z-t}\right) d z \otimes e=\frac{-d w}{2 w} \otimes e .
$$

Then $\pi_{*}(\mathcal{L}, \nabla) \otimes T$ has local exponents $1 / 4,-1 / 4$ at each singular point. Moreover this rank 2 bundle is free if $\mathcal{L} \neq O_{E}$ and has the form $O(1) \oplus O(-1)$ in case $\mathcal{L}=O_{E}$. According to Proposition 2.1 we obtain in this way all the irreducible connections on $\mathbb{P}_{\mathbb{C}}^{1}$ with 4 singular points $0,1, t, \infty$ and all local exponents $1 / 4,-1 / 4$ as push forward of a connection $\nabla e=\omega \otimes e$ on $E$, where $\omega$ is given in standard form by $\left(c+\frac{w+w_{0}}{z-z_{0}}\right) \frac{d z}{2 w}-\frac{d w}{2 w}$ or $c \frac{d z}{2 w}-\frac{d w}{2 w}$, with $c$ a constant and $\left(z_{0}, w_{0}\right) \in E$ (distinct from $\left.1_{E}\right)$. We note that the term $-\frac{d w}{2 w}$ takes care of the required shift of the local exponents. The second standard form for $\omega$ can be seen as a limit case for the first one where $\left(z_{0}, w_{0}\right)$ tends to $1_{E}$. This construction produces also four reducible connections on $\mathbb{P}_{\mathbb{C}}^{1}$, namely for the $\omega$ such that $2 \omega=\frac{d F}{F}$ for some rational function $F$ on $E$. The four cases are

$$
\omega=\frac{w}{z} \frac{d z}{2 w}-\frac{d w}{2 w}, \quad \frac{w}{z-1} \frac{d z}{2 w}-\frac{d w}{2 w}, \quad \frac{w}{z-t} \frac{d z}{2 w}-\frac{d w}{2 w}, \quad-\frac{d w}{2 w} .
$$


Moreover every irreducible connection on $\mathbb{P}_{\mathbb{C}}^{1}$ is obtained precisely twice since $\omega$ and $-\omega$ produce the same connection. These statements easily follow from Observations 2.3. Indeed, the above construction $(\mathcal{L}, \nabla) \mapsto$ $\pi_{*}(\mathcal{L}, \nabla) \otimes T$ translates into the construction, explained in Observations 2.3 , which associates to $\chi \in \operatorname{Hom}\left(\pi_{1}(E), \mathbb{C}^{*}\right)$ a tuple $\left(B_{1}, \ldots, B_{4}\right)$ (with the properties stated above) modulo the action by conjugation of the group $\mathrm{PSL}_{2}(\mathbb{C})$.

\section{The ANALYtic UniVersal FAMILY}

The Legendre family Legendre of elliptic curves $w^{2}=z(z-1)(z-t)$ over $\mathbb{C}$ can be written as algebraic variety

$$
\operatorname{Proj}\left(\mathbb{C}\left[t, \frac{1}{t(t-1)}\right][z, w, s] /\left(s w^{2}-z(z-s)(z-s t)\right)\right) \rightarrow \mathbb{P}^{1} \backslash\{0,1, \infty\} .
$$

If one allows the values $0,1, \infty$ for $t$, then one obtains an elliptic surface $\mathcal{E} \rightarrow \mathbb{P}^{1}$ having singular fibres above $0,1, \infty$. The uniformization of the Legendre family is equal to $\mathbb{H} \times \mathbb{C}_{u}$, where $\mathbb{H}$ is the upper half plane and $\mathbb{C}_{u}$ means $\mathbb{C}$ where we use $u$ as variable. The group acting upon this space is $\mathbb{Z}^{2} \rtimes \Gamma(2)^{*}$, where: $\Gamma(2)^{*}$ is the subgroup of $\mathrm{Sl}_{2}(\mathbb{Z})$ consisting of the matrices $\left(\begin{array}{ll}a & b \\ c & d\end{array}\right)$ such that $a, d \equiv 1 \bmod 4$ and $b, c \equiv 0 \bmod 2$. This group is free on two generators and $\Gamma(2)^{*} \backslash \mathbb{H}$ is isomorphic to $\mathbb{P}^{1} \backslash\{0,1, \infty\}$.

The action (by conjugation) of $\Gamma(2)^{*}$ on $\mathbb{Z}^{2}$ is given by $\gamma\left(\begin{array}{c}n \\ m\end{array}\right) \gamma^{-1}={ }^{t}$ $\gamma^{-1}\left(\begin{array}{c}n \\ m\end{array}\right)$.

The action of $\gamma=\left(\begin{array}{ll}a & b \\ c & d\end{array}\right)$ on $\mathbb{H} \times \mathbb{C}_{u}$ is given by $\gamma(\tau, u)=\left(\frac{a \tau+b}{c \tau+d}, \frac{u}{c \tau+d}\right)$. The action of $\mathbb{Z}^{2}$ is given by $\left(\begin{array}{c}n \\ m\end{array}\right)(\tau, u)=(\tau, u+n \tau+m)$.

Above the Legendre family we want to construct the universal line bundle with a regular connection. Put $\mathbb{T}=\operatorname{Hom}\left(\mathbb{Z}^{2}, \mathbb{C}^{*}\right)$; this is an algebraic torus. Consider the product $\mathbb{T} \times \mathbb{H} \times \mathbb{C}_{u} \times \mathbb{C}_{v}$, where the last term means $\mathbb{C}$ with parameter $v$. This is seen as a geometric line bundle with trivial connection above $\mathbb{T} \times \mathbb{H} \times \mathbb{C}_{u}$. The group $\mathbb{Z}^{2} \rtimes \Gamma(2)^{*}$ act as follows:

For $\gamma=\left(\begin{array}{l}a \\ c \\ c\end{array}\right) \in \Gamma(2)^{*}$, one defines $\gamma(\rho, \tau, u, v)=\left(\gamma(\rho), \frac{a \tau+b}{c \tau+d}, \frac{u}{c \tau+d}, v\right)$, where the action of $\Gamma(2)^{*}$ on $\mathbb{T}$ is induced by its action on $\mathbb{Z}^{2}$. Further, for $\left(\begin{array}{l}n \\ m\end{array}\right) \in \mathbb{Z}^{2}$ one defines $\left(\begin{array}{l}n \\ m\end{array}\right)(\rho, \tau, u, v)=\left(\rho, \tau, u+n \tau+m, \rho\left(\left(\begin{array}{l}n \\ m\end{array}\right)\right) v\right)$. The quotient $\mathbb{L}:=\mathbb{Z}^{2} \rtimes \Gamma(2)^{*} \backslash\left(\mathbb{T} \times \mathbb{H} \times \mathbb{C}_{u} \times \mathbb{C}_{v}\right)$ is a geometric line bundle with a regular connection above the family of elliptic curves $\mathbb{Z}^{2} \rtimes \Gamma(2)^{*} \backslash\left(\mathbb{T} \times \mathbb{H} \times \mathbb{C}_{u}\right)$ parametrized by Par $:=\Gamma(2)^{*} \backslash(\mathbb{T} \times \mathbb{H})$. Let $\mathcal{L}$ be the sheaf of sections of $\mathbb{L}$. It has an induced connection and thus we find a universal regular connection $(\mathcal{L}, \nabla)$ above the family of elliptic curves. The parameter space $\operatorname{Par}:=\Gamma(2)^{*} \backslash \mathbb{T} \times \mathbb{H}$ is a $\mathbb{T}$-bundle above 
$\Gamma(2)^{*} \backslash \mathbb{H}=\mathbb{P}^{1} \backslash\{0,1, \infty\}$. The family of elliptic curves can be written as $\operatorname{Par} \times_{\mathbb{P}^{1} \backslash\{0,1, \infty\}}$ Legendre

Fix $t \in \mathbb{P}^{1} \backslash\{0,1, \infty\}$ and $\tau \in \mathbb{H}$ with image $t$ and a $\rho \in \operatorname{Hom}\left(\mathbb{Z}^{2}, \mathbb{C}^{*}\right)$. The 'evaluation' of $(\mathcal{L}, \nabla)$ at the point $(\rho, t)$ is a connection on the curve $E_{t}=E_{u} /(\mathbb{Z} \tau+\mathbb{Z})$ (with equation $w^{2}=z(z-1)(z-t)$ ) corresponding to the homomorphism $\pi_{1}\left(E_{t}\right)=\mathbb{Z} \tau+\mathbb{Z} \rightarrow \mathbb{C}^{*}$ given by $n \tau+m \mapsto \rho\left(\left(\begin{array}{l}n \\ m\end{array}\right)\right)$.

One considers the subset $\left\{(\rho, \tau) \mid \exists c \in \mathbb{C}\right.$ such that $\left.\rho\left(\left(\begin{array}{c}n \\ m\end{array}\right)\right)=e^{c(n \tau+m)}\right\}$ of $\mathbb{T} \times \mathbb{H}$. This subset is given by the equation $\frac{\log \rho\left(\begin{array}{l}1 \\ 0\end{array}\right)-\tau \log \rho\left(\begin{array}{l}0 \\ 1\end{array}\right)}{2 \pi i} \in \mathbb{Z} \tau+\mathbb{Z}$. (Note that this equation does not depend on the choice of the logarithms). Hence this subset is an analytic divisor on $\mathbb{T} \times \mathbb{H}$. The map $\mathbb{C} \times \mathbb{H} \rightarrow \mathbb{T} \times \mathbb{H}$, given by $(c, \tau) \mapsto(\rho, \tau)$ with $\rho\left(\begin{array}{l}n \\ m\end{array}\right)=e^{c(n \tau+m)}$, induces an isomorphism of $\mathbb{C} \times \mathbb{H}$ with this divisor. The divisor is invariant under the action of $\Gamma(2)^{*}$ and yields a divisor $\Theta$ in the parameter space Par. The subset of the points in the parameter space where the 'evaluation' of $\mathcal{L}$ is trivial, i.e., isomorphic to $O_{E_{t}}$, is precisely $\Theta$. This ends the description of the universal connection of rank one $(\mathcal{L}, \nabla)$ above the space $\operatorname{Par} \times_{\mathbb{P}^{1} \backslash\{0,1, \infty\}}$ Legendre.

The next step is to push this universal connection down to the projective line. One considers the obvious morphism Legendre $\rightarrow$ $\left(\mathbb{P}^{1}-\{0,1, \infty\}\right) \times \mathbb{P}^{1}$. This induces a morphism $\pi: \operatorname{Par} \times_{\mathbb{P}^{1} \backslash\{0,1, \infty\}}$ Legendre $\rightarrow$ Par $\times \mathbb{P}^{1}$. One obtains a connection $\pi_{*}(\mathcal{L}, \nabla)$ of rank 2 on $\operatorname{Par} \times \mathbb{P}^{1}$. This connection is tensorized by the rank 1 connection $(O([\infty]) e, \nabla)$ given by $\nabla e=-\frac{d w}{2 w} \otimes e$. The result is a connection of rank two on the space $\operatorname{Par} \times \mathbb{P}^{1}$ with regular singular sections $\operatorname{Par} \times\{0\}, \ldots, \operatorname{Par} \times\{\infty\}$ and local exponents $1 / 4,-1 / 4$ for each singular section. We delete from $P$ ar the closed subset $\Gamma(2)^{*} \backslash \mathbb{T}[2] \times \mathbb{H}$. On the result $\mathrm{Par}^{*}$ there is a free action of an automorphism of order 2 , induced by the map $\mathbb{T} \times \mathbb{H} \rightarrow \mathbb{T} \times \mathbb{H}$ given by $(\rho, \tau) \mapsto\left(\rho^{-1}, \tau\right)$. The resulting space will de denoted by $\operatorname{Par}^{* *}$. This automorphism also acts upon the connection $\pi_{*}(\mathcal{L}, \nabla) \otimes(O([\infty]) e, \nabla)$ restricted to $\operatorname{Par}^{*} \times \mathbb{P}^{1}$. As a consequence we find a connection $(\mathcal{M}, \nabla)$ on $\operatorname{Par}^{* *} \times \mathbb{P}^{1}$. This is finally the universal family of rank two irreducible connections on $\mathbb{P}^{1}$ with regular singularities at $0,1, t, \infty$ and local exponents $1 / 4,-1 / 4$ at each singular point, that we wanted to construct.

The image $\Theta^{* *}$ of $\Theta$ in $\operatorname{Par}^{* *}$ is the locus where the vector bundle $\mathcal{M}$ is not free (and actually is isomorphic to $O(1) \oplus O(-1)$ ). On the complement of the divisor $\Theta^{* *}$ in $\operatorname{Par}^{* *}$, the vector bundle $\mathcal{M}$ is free. 
The analytic $\mathbb{T}$-bundle $\operatorname{Par} \rightarrow \mathbb{P}^{1} \backslash\{0,1, \infty\}$ is probably not trivial. The same holds for the bundle $\operatorname{Par}^{* *} \rightarrow \mathbb{P}^{1} \backslash\{0,1, \infty\}$. Each fibre is equal to $T \backslash T[2] / \sim$. This space has been identified with $\mathcal{Q} \backslash S$.

The morphism $\Gamma(2)^{*} \backslash(\mathbb{C} \times \mathbb{H}) \rightarrow \mathbb{P}^{1} \backslash\{0,1, \infty\}$ is an analytic line bundle and therefore free. It follows that the divisor $\Theta^{* *}$ on Par $^{* *}$ is, as a variety, isomorphic to the space $\mathbb{C}^{*} \times\left(\mathbb{P}^{1} \backslash\{0,1, \infty\}\right)$. The restriction of the connection $(\mathcal{M}, \nabla)$ to $\Theta^{* *}$ will be called the special family. In Section 6 we will make this special family explicit. The restriction of $\left(\mathcal{M}^{* *}, \nabla\right)$ to $\operatorname{Par}^{* *} \backslash \Theta^{* *}$ has the property that the 'evaluation' of $\mathcal{M}^{* *}$ at every point of this space is free. The connection $\left(\mathcal{M}^{* *}, \nabla\right)$ will be studied from an algebraic point of view in Section 5 .

\section{Algebraic Construction of a moduli family}

The aim is to construct a 'universal' family of irreducible connections $(\mathcal{M}, \nabla)$ on the projective line with $\mathcal{M}$ free, regular singularities at $0,1, t, \infty$ and local exponents $1 / 4,-1 / 4$ at each singular point.

The parameter $t$ is seen as a point in $\mathbb{P}^{1} \backslash\{0,1, \infty\}$. Thus we will work with the projective line over $\mathbb{Q}\left[t, \frac{1}{t(t-1)}\right]$. After fixing a basis of $H^{0}(\mathcal{M})$ and replacing $\nabla$ by $\nabla_{\frac{d}{d z}}$ we obtain a differential operator of the form $\frac{d}{d z}+\sum_{j=1}^{3} \frac{A_{j}}{z-s_{j}}$ with $s_{1}=0, s_{2}=1, s_{3}=t$ and where the matrices $A_{j}=\left(\begin{array}{cc}a_{j} & b_{j} \\ c_{j} & -a_{j}\end{array}\right)$ for $j=1,2,3$ satisfy the conditions: $a_{j}^{2}+b_{j} c_{j}=1 / 16$ for $j=1,2,3$ and $\left(\sum_{j=1}^{3} a_{j}\right)^{2}+\left(\sum_{j=1}^{3} b_{j}\right)\left(\sum_{j=1}^{3} c_{j}\right)=1 / 16$.

We still have to find the conditions which express that the equation is irreducible and moreover we want to divide by the action (by conjugation) of the group $\mathrm{PGl}_{2}$.

The structure of the parameter space.

One considers tuples $\left(A_{1}, \ldots, A_{4}\right)$ of $2 \times 2$-matrices such that $\sum A_{j}=0$ and each $A_{j}$ has eigenvalues $1 / 4,-1 / 4$. The tuples form an affine algebraic variety $\operatorname{Var}$ of dimension 5 , having an action of $\mathrm{PGl}_{2}$, by conjugation. The stabilizer of a tuple $\left(A_{1}, \ldots, A_{4}\right)$ is not trivial if and only if there are two distinct lines invariant under all $A_{j}$. This condition is equivalent to "all $A_{j}$ commute". In this case the stabilizer is the multiplicative group $\mathbb{G}_{m}$. The subset $V a r_{1}$ of $V a r$, consisting of these tuples, is closed; it has dimension 2 and consists of three $\mathrm{PGl}_{2}$-orbits.

The subset $\operatorname{Var}_{2}$ of $V a r$, consisting of the tuples such that the $A_{1}, \ldots, A_{4}$ have a common eigenvector, is also closed. Let $e$ be the 
common eigenvector for all $A_{j}$. Then $A_{j} e= \pm 1 / 4 e$ for all $j$ and the sum of the eigenvalues is 0 . This implies that $\operatorname{Var}_{2}$ has six irreducible components, say $\operatorname{Var}_{2}(i)$ with $i=1, \ldots, 6$, each of dimension 4. Each $\operatorname{Var}_{2}(i)$ is invariant under the action of $\mathrm{PGl}_{2}$. Further the quotient of $\operatorname{Var}_{2}(i) \backslash\left(\operatorname{Var}_{2}(i) \cap \operatorname{Var}_{1}\right)$ by the action of $\mathrm{PGl}_{2}$ is seen to be a projective line.

We are interested in the structure of the quotient $\mathrm{PGl}_{2} \backslash$ Var. There is no geometric quotient. However we will compute the ring $R_{i n v}$ of $\mathrm{PGl}_{2}$-invariant regular functions of $V$ ar. First of all, $A_{4}$ is normalized to $\left(\begin{array}{cc}-1 / 4 & 0 \\ 0 & 1 / 4\end{array}\right)$. This defines a closed subspace $\operatorname{Var}^{\prime}$ of $V a r$. The stabilizer of $A_{4}$, under the action of $\mathrm{PGL}_{2}$ is its maximal torus, isomorphic to the multiplicative group $\mathbb{G}_{m}$. Thus $R_{i n v}$ can be identified with the ring of the regular functions on $V a r^{\prime}$, invariant under $\mathbb{G}_{m}$. The ring of regular functions on $\operatorname{Var}^{\prime}$ is $\mathbb{Q}\left[a_{1}, a_{2}, b_{1}, b_{2}, c_{1}, c_{2}\right]$ with generating relations: $b_{j} c_{j}=1 / 16-a_{j}^{2}$ for $j=1,2$ and $b_{1} c_{2}+b_{2} c_{1}=-2\left(a_{1}-\right.$ $1 / 4)\left(a_{2}-1 / 4\right)$. Using these relations one finds a free basis of the above ring over $\mathbb{Q}\left[a_{1}, a_{2}\right]$ consisting of the monomials $b_{1}^{>0} b_{2}^{\geq 0}, b_{2}^{>0} c_{1}^{\geq 0}, c_{1}^{\geq 0} c_{2}^{\geq 0}$. From this it follows easily that the ring $R_{\text {inv }}$ has the form $\mathbb{Q}\left[a_{1}, a_{2}, H\right]$ with $H=b_{2} c_{1}$. There is only one equation, namely

$$
H^{2}+2 H\left(a_{1}-1 / 4\right)\left(a_{2}-1 / 4\right)+\left(a_{1}^{2}-1 / 16\right)\left(a_{2}^{2}-1 / 16\right)=0 .
$$

We note that also $b_{1} c_{2}$ satisfies this equation. We could have chosen $b_{1} c_{2}$ for $H$, however we prefer $H=b_{2} c_{1}$ in view of later computations. The singular locus of $R_{i n v}$ is equal to

$$
\left(a_{1}, a_{2}, H\right)=(1 / 4,1 / 4,0),(1 / 4,-1 / 4,0),(-1 / 4,1 / 4,0) .
$$

This singular locus is precisely the collection of points where the 4 matrices $A_{1}, \ldots, A_{4}$ have a common eigenvector. Hence the regular locus of $\operatorname{Spec}\left(R_{\text {inv }}\right)$ coincides with the variety $\mathrm{PGl}_{2} \backslash\left(\operatorname{Var}-\operatorname{Var}_{2}\right)$. This space is not affine and for further calculations we consider the open affine subset given by $\left(a_{1}^{2}-1 / 16\right)\left(a_{2}^{2}-1 / 16\right)\left(a_{3}^{2}-1 / 16\right) \neq 0$ (note that $\left.a_{3}=1 / 4-a_{1}-a_{2}\right)$. For the missing points, given by $a_{j}= \pm 1 / 4$ with $j=1,2,3$, we make separate calculations.

The open affine part $U$ of the parameter space $P$.

The complete parameter space $P$ is given by

$$
P:=\operatorname{Spec}\left(\mathbb{Q}\left[t, \frac{1}{t(1-t)}\right]\right) \times\left(\mathrm{PGL}_{2} \backslash\left(\operatorname{Var}-\operatorname{Var}_{2}\right)\right) .
$$

As before we normalize $A_{4}$ and identify the right hand side with the regular locus of $\operatorname{Spec}\left(R_{\text {inv }}\right)$. Further we consider the open affine subset $U$ given by $s \neq 0$ where $s=\left(a_{1}^{2}-1 / 16\right)\left(a_{2}^{2}-1 / 16\right)\left(a_{3}^{2}-1 / 16\right)$. Then $H \neq 0$ and thus $b_{2} c_{1} \neq 0$. We normalize further by $c_{1}=1$. The 
corresponding ring is now $R_{U}:=\mathbb{Q}\left[t, \frac{1}{t(1-t)}\right]\left[a_{1}, a_{2}, \frac{1}{s}, b_{2}\right] /(\operatorname{Rel})$ with Rel $=b_{2}^{2}+2 b_{2}\left(a_{1}-1 / 4\right)\left(a_{2}-1 / 4\right)+\left(a_{1}^{2}-1 / 16\right)\left(a_{2}^{2}-1 / 16\right)$. The other variables are expressed in $a_{1}, a_{2}, b_{2}, 1 / s$ by the formulas

$$
\begin{gathered}
b_{1}=1 / 16-a_{1}^{2} ; c_{2}=b_{2}^{-1}\left(1 / 16-a_{2}^{2}\right) ; a_{3}=1 / 4-a_{1}-a_{2} \\
b_{2}^{-1}=-b_{2} \frac{a_{3}^{2}-1 / 16}{s}-2 \frac{\left(a_{1}-1 / 4\right)\left(a_{2}-1 / 4\right)\left(a_{3}^{2}-1 / 16\right)}{s} \\
b_{3}=-b_{2}-1 / 16+a_{1}^{2} ; c_{3}=-1-b_{2}^{-1}\left(1 / 16-a_{2}^{2}\right)
\end{gathered}
$$

The locus of the reducible equations.

We search the locus $P_{\text {reducible }} \subset P$, consisting of the points in $P(\overline{\mathbb{Q}})$, where the corresponding differential operator $D:=\frac{d}{d z}+\sum_{j=1}^{3} \frac{A_{j}}{z-s_{j}}$ is reducible. A point corresponds to prescribed values in $\overline{\mathbb{Q}}$ for $a_{1}, a_{2}, \ldots, t$. Reducibility is equivalent to the existence of a non zero vector $v \in$ $\overline{\mathbb{Q}}[z] \otimes \mathbb{Q}^{2}$ such that $D(v)=f v$ for some $f \in \overline{\mathbb{Q}}(z)$. The vector $v$ can be normalized such that the g.c.d. in the ring $\overline{\mathbb{Q}}[z]$ of all its coefficients is 1 . Thus one considers $v:=v_{0}+v_{1} z+\cdots+v_{d} z^{d}$, with all $v_{i} \in \overline{\mathbb{Q}}^{2}$, $v_{d} \neq 0$ and $v_{0}+v_{1} s+\cdots+v_{d} s^{d} \neq 0$ for all $s \in \overline{\mathbb{Q}}$, and satisfying the equation $D(v)=f v$ for some $f \in \overline{\mathbb{Q}}(z)$. One easily verifies that $f=\left(\sum_{j=1}^{3} \frac{\lambda_{j}}{z-s_{j}}\right)$ where the $\lambda_{j}$ are eigenvalues of $A_{j}$. Expanding the equation $D(v)=f v$ at $z=\infty$ yields that $d=\sum_{j=1}^{4} \lambda_{j}$, where $\lambda_{4}$ is some eigenvalue of $A_{4}=-\sum_{j=1}^{3} A_{j}$. In this special case there are only two possibilities, namely $d=0$ and $d=1$. In first case all $A_{j}$ have a common eigenvector. This case is excluded by the definition of the parameter space $P$. The case $d=1$ is equivalent to the equation

$$
\left(\frac{d}{d z}+\sum_{j=1}^{3} \frac{A_{j}-1 / 4}{z-s_{j}}\right)\left(v_{0}+v_{1} z\right)=0 .
$$

The normalization of $A_{4}$ implies $v_{1}=e_{2}=\left(\begin{array}{l}0 \\ 1\end{array}\right)$, where $e_{1}, e_{2}$ is the standard basis of $\mathbb{Q}^{2}$. The above equation is equivalent to the system of matrix equations

$$
\left(A_{1}-1 / 4\right) v_{0}=0,\left(A_{2}-1 / 4\right)\left(v_{0}+e_{2}\right)=0,\left(A_{3}-1 / 4\right)\left(v_{0}+t e_{2}\right)=0 .
$$

The existence of a solution $v_{0}$ leads to certain relations between the coefficients of the matrices $A_{1}, A_{2}, A_{3}$, describing in fact $P_{\text {reducible }}$.

The direct approach. Let $T$ denote the affine ring of $V a r^{\prime}$. Recall that $T:=\mathbb{Q}\left[a_{1}, a_{2}, b_{1}, b_{2}, c_{1}, c_{2}\right]$ with generating relations $b_{j} c_{j}=1 / 16-a_{j}^{2}$ for $j=1,2$ and $b_{1} c_{2}+b_{2} c_{1}=-2\left(a_{1}-1 / 4\right)\left(a_{2}-1 / 4\right)$. Consider $T\left[x_{1}, x_{2}\right]$, where $v_{0}=\left(\begin{array}{l}x_{1} \\ x_{2}\end{array}\right)$. The system of matrix equations defines an ideal $I \subset T\left[x_{1}, x_{2}\right]$. Using Gröbner basis one calculates $J=I \cap T$. This 
ideal is invariant under the action by conjugation of $\mathbb{G}_{m}$. Therefore $J$ is induced by an ideal $J_{0}$ of $T^{\mathbb{G}_{m}}=R_{i n v}=\mathbb{Q}\left[t, \frac{1}{t(t-1)}\right]\left[a_{1}, a_{2}, H\right]$ (with generating relation $\mathrm{Rel}=H^{2}+2 H\left(a_{1}-1 / 4\right)\left(a_{2}-1 / 4\right)+\left(a_{1}^{2}-1 / 16\right)\left(a_{2}^{2}-\right.$ $1 / 16)=0)$ in the sense that $J=J_{0} T$. The ideal $J_{0}$ turns out to be generated by the two elements

$$
\begin{gathered}
f(t)=\left(a_{1}+a_{2}-1 / 2\right)^{2} t(t-1)+\left(a_{1}+1 / 4\right)^{2} t+\left(a_{2}+1 / 4\right)^{2}(1-t) \text { and } \\
2 H+\left(a_{1}+a_{2}-1 / 2\right)^{2} t+\left(a_{2}+1 / 4\right)\left(a_{1}-a_{2}\right) .
\end{gathered}
$$

One computes that the ideal $I$ also contains the elements

$$
x_{1}+2 b_{1} t+2 b_{2} t-2 b_{2} \text { and } x_{2}+1 / 2 t-a_{1} t-a_{2} t+a_{2}+1 / 4 .
$$

Thus $T\left[x_{1}, x_{2}\right] / I=T / J$ and $(T / J)^{\mathbb{G}_{m}}=R_{\text {inv }} / J_{0}$. The last algebra equals $\mathbb{Q}\left[t, \frac{1}{t(t-1)}\right]\left[a_{1}, a_{2}\right] /(f(t))$. One easily verifies that $f(t)$ is irreducible. Therefore $\mathbb{Q}\left[t, \frac{1}{t(t-1)}\right]\left[a_{1}, a_{2}\right] /(f(t))$ is a domain of dimension 2. Thus $J_{0} \subset R_{\text {inv }}$ is a prime ideal of height 1 and defines a closed irreducible subset of $\operatorname{Spec}\left(R_{i n v}\right)$ of codimension 1. The intersection of this closed subset with $P$ is $P_{\text {irreducible }}$ and the latter is therefore closed and irreducible of dimension 2. Moreover $P_{\text {irreducible }}$ has the following rational parametrization by $t, \lambda$

$$
a_{1}=\frac{t-2 \lambda^{2}}{4 t}, a_{2}=\frac{t+1+2 \lambda^{2}+4 \lambda}{4(t-1)}, H=\frac{(\lambda+1) \lambda\left(\lambda^{2}-t\right)}{4 t(t-1)},
$$

with $\lambda=t a_{1}+t a_{2}-t / 2-a_{2}-1 / 4$.

\section{The SPeCial FAmily AND the Lamé EQUATion}

The 'special family' that we compute here is the family of irreducible connections $(\mathcal{M}, \nabla)$ on $\mathbb{P}^{1}$ with (as before) 4 regular singular points at $0,1, t, \infty$, local exponents $1 / 4,-1 / 4$ at each singular point and such that $\mathcal{M}$ is not free. We note that for a suitable shift of the local exponents over integers the connection can be presented with a free vector bundle.

The second exterior power $\Lambda^{2}(\mathcal{M}, \nabla)$ is a rank one connection without singularities on $\mathbb{P}^{1}$. Hence $\Lambda^{2} \mathcal{M}$ is the trivial line bundle and the connection on it is also trivial. The irreducibility of $(\mathcal{M}, \nabla)$ implies that $\mathcal{M}$ is isomorphic to the sheaf $O(1) \oplus O(-1)$. Using this information one can calculate the a matrix form for the connection. Instead of producing the results of this calculation, we will describe an easier method to obtain the special family.

Proposition 3.2 provides another way to obtain this family. On the elliptic curve $E$, given by $w^{2}=z(z-1)(z-t)$, one considers the connection $\nabla: O_{E} f_{0} \rightarrow \Omega \otimes O_{E} f_{0}$ given by $\nabla\left(f_{0}\right)=c \frac{d z}{2 w} \otimes f_{0}$ with 
$c \neq 0$. The direct image, under the morphism $E \rightarrow \mathbb{P}^{1}$ has w.r.t. the basis $f_{0}, f_{1}=w f_{0}$ the matrix form

$$
\frac{d}{d z}+\left(\begin{array}{cc}
0 & c / 2 \\
\frac{c}{2 w^{2}} & \frac{w^{\prime}}{w}
\end{array}\right)
$$

One has to shift over $-\frac{w^{\prime}}{2 w}$ in order to obtain the local exponents $1 / 4,-1 / 4$. After a conjugation with the constant matrix $\left(\begin{array}{cc}2 c^{-1} & 0 \\ 0 & 1\end{array}\right)$ one obtains the required form (with $d=c^{2} / 4$ )

$$
\frac{d}{d z}+\left(\begin{array}{cc}
-\frac{w^{\prime}}{2 w} & d \\
\frac{1}{w^{2}} & \frac{w^{\prime}}{2 w}
\end{array}\right) .
$$

The above is in fact a family over the base ring $\mathbb{Q}\left[t, \frac{1}{t(t-1)}, d, d^{-1}\right]$.

The classical Lamé equation is $\mathcal{L}_{n}:=y^{\prime \prime}+\frac{f^{\prime}}{2 f} y^{\prime}-\frac{n(n+1) z+B}{f} y$, with $f=4\left(z^{3}+a z+b\right)$. The transformation $z \mapsto z-\frac{1+t}{3}$, with suitable $t$, transforms $f$ into $4 z(z-1)(z-t)$ and the equation into

$$
y^{\prime \prime}+\frac{g^{\prime}}{2 g} y^{\prime}-\frac{n(n+1)(z-(1+t) / 3)+B}{4 g} y \text { with } g=z(z-1)(z-t) .
$$

The local exponents are $0,1 / 2$ for $0,1, t$ and $-n / 2,(n+1) / 2$ at $\infty$. For $n=0$ one writes $w^{2}=g$. The companion matrix equation for the second scalar equation is $\frac{d}{d z}+\left[\begin{array}{cc}0 & -1 \\ \frac{-B}{4 w^{2}} & \frac{w^{\prime}}{w}\end{array}\right]$. The shift yields the system $\frac{d}{d z}+\left[\begin{array}{cc}-\frac{w^{\prime}}{2 w} & -1 \\ \frac{-B}{4 w^{2}} & \frac{w^{\prime}}{2 w}\end{array}\right]$.

If $B \neq 0$, then conjugation with a constant matrix yields the equation $\frac{d}{d z}+\left[\begin{array}{cc}-\frac{w^{\prime}}{2 w} & \frac{4}{B} \\ \frac{1}{w^{2}} & \frac{w^{\prime}}{2 w}\end{array}\right]$. This is the special family.

The classical Lamé equation with $n=1$ is gauge equivalent to the sub-family of Lamé systems defined by $a_{1}=1 / 4$ and $H=0$; the parameter $B=\left(e_{2}-e_{1}\right) b$ is therefore given by $b=2(1-t) a_{2}+\frac{t+1}{6}$. It is known that $D_{2}^{S L_{2}}$ does not occur as a Galois group Lamé equation for $n=1$ (see [4]) and one can verify that the previous family does not intersect the irreducible component of $P_{2}$ described above for $t \neq$ $0,1, \infty$. It is also known that $D_{3}^{S L_{2}}$ occurs once as a Galois group for $n=1$ (see [5]) and can be deduced from other components of $P_{3}$ given by order 6 points on $E_{t}$ : we find $B=0$ and $t^{2}-t+1=0$ which gives one solution up to Moebius transformation of $z$. 
The classical Lamé equation with $n=2$ is gauge equivalent to the sub-family of Lamé systems defined by

$$
\begin{gathered}
16(t-1)^{2}\left(4 a_{1}+7\right) a_{2}^{2}+8(t-1)\left(4 a_{1}-1\right)\left(4 t a_{1}+4 t-3\right) a_{2} \\
+\left(64 t^{2} a_{1}^{3}-16 t(t-2) a_{1}^{2}-4\left(8 t^{2}-14 t+7\right) a_{1}-1\right)=0, \\
H=\frac{\left(a_{1}-\frac{1}{4}\right)\left(t a_{1}+(1-t) a_{2}+\frac{2 t-1}{4}\right)}{2(t-1)}, \\
\text { and } b=\frac{4(t-2) a_{1}+12(t-1) a_{2}-2 t+1}{4 a_{1}+1} .
\end{gathered}
$$

Here, $D_{2}^{S L_{2}}$ occurs twice as Galois group exactly through the irreducible component of $P_{2}$ presented above: we find $t=-1$ and $b^{2}=\frac{72}{7}$. There are many occurencies of $D_{3}^{S L_{2}}$ as Galois group, most of them arising from points of order 6 on $E_{t}$; the contribution of the component of $P_{3}$ defined by the points of order 3 on $E_{t}$ is given by:

$$
t+1=0 \quad \text { and } \quad 359 b^{4}-7128 b^{2}+34992=0
$$

and

$$
125 t^{2}-234 t+125=0 \text { and } \quad b=-\frac{7(t+1)}{11} .
$$

Finally, it follows from [6] that the smooth compactification over $t=$ $0,1, \infty$ of the locus $P_{N}$ identify with the modular curve $X_{00}(N, 2)$ studied in [1]: it is irreducible for $N$ odd and has three irreducible components for $N$ even; the genus of $P_{N}$ for $N=p>2$ prime is $\frac{1}{4}(p-3)^{2}$.

\section{Explicit formulas AND Painlevé VI}

7.1. An inverse approach. The family $\{(\mathcal{M}, \nabla)\}$ can be computed directly from the normalized differential form $\omega=\left(c+\frac{w+w_{0}}{z-z_{0}}\right) \frac{d z}{2 w}$ of Section 3. The differential module above the elliptic curve reads $\partial f_{0}=$ $\left(c+\frac{w+w_{0}}{z-z_{0}}\right) \frac{1}{2 w} f_{0}$. With respect to the basis $f_{0}, f_{1}=w f_{0}$ over the field of rational functions on $\mathbb{P}^{1}$ one finds the operator

$$
\begin{gathered}
\frac{d}{d z}+\left[\begin{array}{cc}
\frac{1}{2\left(z-z_{0}\right)} & \left(c+\frac{w_{0}}{\left(z-z_{0}\right)}\right) / 2 \\
\frac{1}{2 w^{2}}\left(c+\frac{w_{0}}{\left(z-z_{0}\right)}\right) & \frac{w^{\prime}}{w}+\frac{1}{2\left(z-z_{0}\right)}
\end{array}\right] . \text { The shift over }-\frac{w^{\prime}}{2 w} \text { yields } \\
L_{0}:=\frac{d}{d z}+\left[\begin{array}{cc}
\frac{1}{2\left(z-z_{0}\right)}-\frac{w^{\prime}}{2 w} & \left(c+\frac{w_{0}}{\left(z-z_{0}\right)}\right) / 2 \\
\frac{1}{2 w^{2}}\left(c+\frac{w_{0}}{\left(z-z_{0}\right)}\right) & \frac{w^{\prime}}{2 w}+\frac{1}{2\left(z-z_{0}\right)}
\end{array}\right] . \text { This is replaced by } \\
L_{1}:=\left(\begin{array}{cc}
1 & -c z \\
0 & 1
\end{array}\right) L_{0}\left(\begin{array}{cc}
1 & c z \\
0 & 1
\end{array}\right) \text { in order to remove the apparent pole }
\end{gathered}
$$
of order 2 at $\infty$. 
The formulas for $w_{0} \neq 0$ (equivalently $z_{0} \neq 0,1, t$ ).

$z_{0}$ is an apparent singularity of $L_{1}$ which can be removed as follows. First calculate the conjugate

$$
\begin{gathered}
L_{2}:=\left(\begin{array}{cc}
\frac{1}{2 w_{0}} & -1 / 2 \\
\frac{1}{2 w_{0}} & 1 / 2
\end{array}\right) L_{1}\left(\begin{array}{cc}
w_{0} & w_{0} \\
-1 & 1
\end{array}\right) \text {. Then again a conjugate } \\
L_{3}:=\left(\begin{array}{cc}
1 & 0 \\
0 & z-z_{0}
\end{array}\right) L_{2}\left(\begin{array}{cc}
1 & 0 \\
0 & \left(z-z_{0}\right)^{-1}
\end{array}\right) .
\end{gathered}
$$

Then $L_{3}$ is conjugated by $\left(\begin{array}{ll}0 & 1 \\ 1 & 0\end{array}\right)$ in order to normalize the matrix at infinity to $\left(\begin{array}{cc}-1 / 4 & 0 \\ 0 & 1 / 4\end{array}\right)$. Finally, a conjugation with a constant matrix of the form $\left(\begin{array}{ll}1 & 0 \\ 0 & s\end{array}\right)$ is needed to obtain $c_{1}=1$. The final operator $\frac{d}{d z}+U$ belongs to our family, normalized with $c_{1}=1$.

$$
U=\frac{1}{\Delta}\left[\begin{array}{cc}
N & \frac{\left(c z-c z_{0}+w_{0}\right) F}{t} \\
4 t-4 z t \frac{2 w_{0}-3 c z_{0}+c+c t+c^{3}}{F} & -N
\end{array}\right]
$$

where

$$
\begin{gathered}
\Delta=4 z(z-1)(z-t) \\
N=z^{2}+\left(2 c^{2}-2 z_{0}\right) z+2 z_{0} t-t-2 c^{2} z_{0}-2 z_{0}^{2}+2 z_{0} \\
F=3 c z_{0}^{2}-3 c z_{0} t-3 c z_{0}-w_{0} z_{0}+c^{3} z_{0}+2 c t-3 c^{2} w_{0}+w_{0} t+w_{0}
\end{gathered}
$$

\section{Remarks 7.1.}

(1) This formula for $U$ is derived under the assumption that $w_{0} \neq 0$. The normalization $c_{1}=1$ introduces the denominator $F$ in the $(2,1)$ entry of $U$. Thus the formula for $U$ is valid under the assumptions that $w_{0} \neq 0$ and $F \neq 0$. The involution of the elliptic curve maps $\left(c, w_{0}\right)$ to $\left(-c,-w_{0}\right)$. One observes that the formula for $U$ is, as it should be, invariant under this map.

(2) Suppose again that $w_{0} \neq 0$ and $F \neq 0$. Conjugation of $U$ with the constant matrix $\left(\begin{array}{ll}t & 0 \\ 0 & F\end{array}\right)$ yields

$$
U^{*}=\frac{1}{\Delta}\left[\begin{array}{cc}
N & c z-c z_{0}+w_{0} \\
-4 z\left(-3 c z_{0}+2 w_{0}+c+c t+c^{3}\right)+4 F & -N
\end{array}\right]
$$

Thus $\frac{d}{d z}+U$ is equivalent to $\frac{d}{d z}+U^{*}$. The latter expression is also valid for $F=0$. We will show that the formula $\frac{d}{d z}+U^{*}$ remains valid for $w_{0}=0$. Another advantage of $U^{*}$ is that its $(1,2)$ entry has a zero if $c \neq 0$. We note that $U^{*}$ is not invariant under the involution of the elliptic curve. 
The formulas for $w_{0}=0$ (equivalently $z_{0} \in\{0,1, t\}$ ).

As before $L_{0}:=\frac{d}{d z}+\left[\begin{array}{cc}\frac{1}{2\left(z-z_{0}\right)}-\frac{w^{\prime}}{2 w} & c / 2 \\ \frac{c}{2 w^{2}} & \frac{w^{\prime}}{2 w}+\frac{1}{2\left(z-z_{0}\right)}\end{array}\right]$ and $L_{1}$ is obtained by conjugating $L_{0}$ with $\left(\begin{array}{cc}1 & c\left(z-z_{0}\right) \\ 0 & 1\end{array}\right)$. Further $L_{2}$ is obtained from $L_{1}$ by conjugation with $\left(\begin{array}{cc}1 & 0 \\ 0 & \left(z-z_{0}\right)^{-1}\end{array}\right)$. Finally $L_{3}$ is obtained from $L_{2}$ by conjugation with $\left(\begin{array}{ll}0 & 1 \\ 1 & 0\end{array}\right)$. We will not further normalize $L_{3}$. One finds the following formulas.

(1) For $z_{0}=0, L_{3}=\frac{d}{d z}+U_{0}$ with

$$
U_{0}=\frac{1}{\Delta}\left[\begin{array}{cc}
z^{2}-t+2 c^{2} z & c z \\
4 c\left(\left(-1-t-c^{2}\right) z+2 t\right) & -z^{2}+t-2 c^{2} z
\end{array}\right]
$$

(2) For $z_{0}=1, L_{3}=\frac{d}{d z}+U_{1}$ with

$$
U_{1}=\frac{1}{\Delta}\left[\begin{array}{cc}
z^{2}+2\left(c^{2}-1\right) z-2 c^{2}+t & c(z-1) \\
4 c^{3}-4 c t-4 z\left(c t-2 c+c^{3}\right) & 2 c^{2}-t-2\left(c^{2}-1\right) z-z^{2}
\end{array}\right]
$$

(3) For $z_{0}=t, L_{3}=\frac{d}{d z}+U_{t}$ where $U_{t}$ equals

$$
\frac{1}{\Delta}\left[\begin{array}{cc}
z^{2}+\left(2 c^{2}-2 t\right) z-t\left(2 c^{2}-1\right) & c(z-t) \\
-4 z\left(c-2 c t+c^{3}\right)+4 c^{3} t-4 c t & t\left(2 c^{2}-1\right)+\left(2 t-2 c^{2}\right) z-z^{2}
\end{array}\right]
$$

These formulas are specializations of $U^{*}$ for $w_{0}=0$ and $z_{0}=0,1, t$.

7.2. A direct approach. On the space $P \backslash P_{\text {reducible, }}$, the matrix $U$ (or $U^{*}$ ) expresses $a_{1}, a_{2}, b_{2}, t$ in terms of $z_{0}, w_{0}, c$. The aim is to express the rational functions $z_{0}, \frac{w_{0}}{c}, c^{2}$ on this space in terms of $a_{1}, a_{2}, b_{2}$. One can view the data of $U$ and $U^{*}$ as polynomials $a_{1}, a_{2}, b_{2}, z_{0}, w_{0}, c, t$, generating an ideal in $\mathbb{Q}\left[a_{1}, a_{2}, b_{2}, t, \frac{1}{t(t-1)}, z_{0}, w_{0}, c\right]\left(b_{2}\right.$ stands for $H$ and there are known relations for $a_{1}, a_{2}, b_{2}$ and $\left.z_{0}, w_{0}, c\right)$. Elimination for a suitable order produces two elements in this ideal, namely

$\left(16 t^{2} a_{1}^{2}-32 a_{2}^{2} t+32 a_{1} a_{2} t^{2}-32 a_{1} a_{2} t-16 t^{2} a_{1}+24 a_{1} t+16 a_{2}^{2}+16 a_{2}^{2} t^{2}+8 a_{2}-16 a_{2} t^{2}+8 a_{2} t+1+4 t^{2}-4 t\right) z_{0}$

$-t\left(-3+48 a_{1} a_{2} t+20 a_{1}-20 a_{2} t+20 a_{2}-12 a_{1} t-48 a_{2} a_{1}+4 t+16 t a_{1}{ }^{2}+32 t b_{2}-32 b_{2}\right)$

and

$\left((-32+32 t) b_{2}-\left(1+4 a_{1}\right)\left(4 a_{1} t-4 a_{2} t+1+4 a_{2}\right)\right) z_{0}+t\left(1+4 a_{1}\right)^{2}$.

We recall that the locus $P_{\text {reducible }}$ was given by an ideal $\left(E q_{1}, E q_{2}\right)$, given by

$$
\begin{gathered}
E q_{1}=\left(a_{1}+a_{2}-1 / 2\right)^{2} t(t-1)+\left(a_{1}+1 / 4\right)^{2} t+\left(a_{2}+1 / 4\right)^{2}(-t+1) \\
E q_{2}=2 b_{2}+\left(a_{1}+a_{2}-1 / 2\right)^{2} t+\left(a_{2}+1 / 4\right)\left(a_{1}-a_{2}\right)
\end{gathered}
$$


The coefficients $P_{1}, P_{2}$ of $z_{0}$ in the above equations satisfy $P_{1}=16 \cdot E q_{1}$ and $P_{2}=16(t-1) \cdot E q_{2}-16 \cdot E q_{1}$. Thus $\left(P_{1}, P_{2}\right)=\left(E q_{1}, E q_{2}\right)$. The locus $P \backslash P_{\text {reducible }}$ is the union of the two open subsets of $P$ given by $P_{1} \neq 0$ and $P_{2} \neq 0$. On each one of them, $z_{0}$ is a regular expression in $a_{1}, a_{2}, b_{2}, t$. For $P_{2} \neq 0$ this expression is

$$
z_{0}=-\frac{t\left(1+4 a_{1}\right)^{2}}{32(t-1) b_{2}-\left(1+4 a_{1}\right)\left(4 a_{1} t-4 a_{2}(t-1)+1\right)} .
$$

Once $z_{0}$ is known, we may choose a solution $w_{0}$ of $w_{0}^{2}=z_{0}\left(z_{0}-1\right)\left(z_{0}-t\right)$. For the zero $q$ of the $(1,2)$ coefficient of $U$ we have two expression, namely $q=z_{0}-\frac{w_{0}}{c}$ and $q=-\frac{t\left(16 a_{1}^{2}-1\right)}{16(t-1) b_{2}-t\left(16 a_{1}^{2}-1\right)}$. This yields a formula for $c$. We recall that the pair $\left(w_{0}, c\right)$ is unique up to a sign.

7.3. Intermezzo on Painlevé VI. We consider a family of connections, more general than the Lamé connection,

$$
\frac{d}{d z}+A=\frac{d}{d z}+\frac{A_{1}}{z}+\frac{A_{2}}{z-1}+\frac{A_{3}}{z-t} \text { with } A_{j}=\left(\begin{array}{cc}
a_{j} & b_{j} \\
c_{j} & -a_{j}
\end{array}\right) \text { for } j=1,2,3 \text {. }
$$

Put $A_{\infty}=-A_{1}-A_{2}-A_{3}$. The assumptions are:

$A_{j}$ has eigenvalues $\pm \frac{\theta_{j}}{2}$ (equivalently $a_{j}^{2}+b_{j} c_{j}=\frac{\theta_{j}^{2}}{4}$ for $j=1,2,3$ ),

$A_{\infty}=\left(\begin{array}{cc}-\frac{\theta_{\infty}}{2} & 0 \\ 0 & \frac{\theta_{\infty}}{2}\end{array}\right)$ (equivalently $\sum a_{j}=\frac{\theta_{\infty}}{2}, \sum b_{j}=0, \sum c_{j}=0$ ).

The $(1,2)$ entry of $A$ has a single zero, namely $q:=\frac{b_{1} t}{b_{1} t+b_{2}(t-1)}$ (if $b_{1} t+b_{2}(t-1)=0$ then we write $\left.q=\infty\right)$. One considers in this space of connections a family $\frac{d}{d z}+A(t)$ (i.e., all $a_{j}, b_{j}, q, \ldots$ are analytic functions of $t$ ). Fuchs theorem can be formulated as follows (compare $[\mathrm{J}-\mathrm{M}]$ ).

Theorem 7.2 (Fuchs). Suppose that $\theta_{j} \notin \mathbb{Z}$ for $j=1,2,3, \infty$. Consider family $\frac{d}{d z}+A(t)$, holomorphic in $t$ and defined in a neighbourhood of $t_{0}$. Suppose that not all $A_{j}\left(t_{0}\right)$ commute and that $q\left(t_{0}\right) \neq \infty$. Then the family is isomonodromic if and only if $q=q(t)$ satisfies

$$
q^{\prime}=2 a_{1} \frac{q-1}{t-1}+2 a_{2} \frac{q}{t}+\left(1-\theta_{\infty}\right) \frac{q(q-1)}{t(t-1)}
$$

and the Painlevé VI equation

$$
\begin{gathered}
q^{\prime \prime}=1 / 2\left(\frac{1}{q}+\frac{1}{q-1}+\frac{1}{q-t}\right)\left(q^{\prime}\right)^{2}-\left(\frac{1}{t}+\frac{1}{t-1}+\frac{1}{q-t}\right) q^{\prime}+ \\
\frac{q(q-1)(q-t)}{t^{2}(t-1)^{2}}\left(\alpha+\beta \frac{t}{q^{2}}+\gamma \frac{t-1}{(q-1)^{2}}-\delta \frac{t(t-1)}{(q-t)^{2}}\right) .
\end{gathered}
$$

with special parameters $(\alpha, \beta, \gamma, \delta)=\left(\frac{\left(\theta_{\infty}-1\right)^{2}}{2},-\frac{\theta_{1}^{2}}{2}, \frac{\theta_{2}^{2}}{2}, \frac{\theta_{3}^{2}-1}{2}\right)$. 
If $q$ is known, then one recovers all $a_{j}, b_{j}, c_{j}$ (up to the action of $\mathbb{G}_{m}$ on the $b_{j}$ and $c_{j}$ ) from the equality $q:=\frac{b_{1} t}{b_{1} t+b_{2}(t-1)}$ and the equation for $q^{\prime}$. The choice $\theta_{j}=1 / 2$ for $j=1,2,3, \infty$ defines the Lamé connection.

A point of order $m$ on the elliptic curve $w^{2}=z(z-1)(z-t)$ can be seen as a pair $\left(z_{0}(t), w_{0}(t)\right)$ of algebraic functions in $t$. It has been shown by É. Picard, [Pic], that the coordinate $z_{0}(t)$ is a solution of PVI $\left(0,0,0, \frac{1}{2}\right)$ (compare [Maz]).

We will use the transformation $w_{1} w_{2} w_{1}$ of Okamoto to obtain solutions for PVI $\left(\frac{1}{8},-\frac{1}{8}, \frac{1}{8}, \frac{3}{8}\right)$ corresponding to Lamé parameters. This transformation is described as follows (see [Ok, p. 356]).

Suppose that $q$ is a solution of PVI for the parameters $\left(\theta_{1}, \theta_{2}, \theta_{3}, \theta_{\infty}\right)$. Then $q+\frac{1}{2 p}$ with

$$
p=\frac{t(t-1) q^{\prime}}{2 q(q-1)(q-t)}+\frac{\theta_{1}}{2 q}+\frac{\theta_{2}}{2(q-1)}+\frac{\theta_{3}-1}{2(q-t)}
$$

is a solution of PVI for the parameters

$$
\begin{gathered}
\theta_{1}^{*}=1 / 2\left(\theta_{1}-\theta_{2}+\theta_{3}+\theta_{\infty}\right)-1, \theta_{2}^{*}=1 / 2\left(-\theta_{1}+\theta_{2}+\theta_{3}+\theta_{\infty}\right)-1, \\
\theta_{3}^{*}=1 / 2\left(\theta_{1}+\theta_{2}+\theta_{3}-\theta_{\infty}\right)+1, \theta_{\infty}^{*}=1 / 2\left(\theta_{1}+\theta_{2}-\theta_{3}+\theta_{\infty}\right)+1 .
\end{gathered}
$$

In particular, a solution $q$ of PVI $\left(0,0,0, \frac{1}{2}\right)$ yields a solution $q+\frac{1}{2 p}$, with $p=\frac{t(t-1) q^{\prime}}{2 q(q-1)(q-t)}-\frac{1}{2(q-t)}$ with $q^{\prime}=\frac{d q}{d t}$ is a solution of PVI $\left(\frac{1}{8},-\frac{1}{8}, \frac{1}{8}, \frac{3}{8}\right)$.

In particular, a point $\left(z_{0}(t), w_{0}(t)\right)$ of order $m$ on the elliptic curve yields the algebraic solution $z_{0}-\frac{z_{0}\left(z_{0}-1\right)\left(z_{0}-t\right)}{z_{0}\left(z_{0}-1\right)-t(t-1) z_{0}^{\prime}}\left(\right.$ with $z_{0}=z_{0}(t)$ and $\left.z_{0}^{\prime}=\frac{d z_{0}}{d t}\right)$ for PVI $\left(\frac{1}{8},-\frac{1}{8}, \frac{1}{8}, \frac{3}{8}\right)$.

7.4. Points of finite order on the elliptic curve. Each member $\nabla_{*}$ of the Lamé family is induced by a regular equation of rank one on the elliptic curve $E$, given by $w^{2}=z(z-1)(z-t)$. Taking the shift into account, $\nabla_{*}$ is the push forward of $\nabla e=\left\{\left(c+\frac{w+w_{0}}{z-z_{0}}\right) \frac{d z}{2 w}-\frac{d w}{2 w}\right\} \otimes e$ or of $\nabla e=\left\{c \frac{d z}{2 w}-\frac{d w}{2 w}\right\} \otimes e$, where $c$ a constant and $\left(z_{0}, w_{0}\right) \in E$.

For the moment we fix a complex value for $t$ (different from $0,1, \infty$ ) and take $\left(z_{0}, w_{0}\right) \in E(\mathbb{C})$. The differential Galois group of $\nabla_{*}$ is equal to $D_{n}^{\mathrm{SL}_{2}}$ if and only if the corresponding rank one equation on $E$ has a cyclic differential Galois group of order $2 n$. The latter is equivalent to the statement that the equation $\nabla e=\left(c+\frac{w+w_{0}}{z-z_{0}}\right) \frac{d z}{2 w} \otimes e$ or $\nabla e=c \frac{d z}{2 w} \otimes e$, has a cyclic differential Galois of order $m$ such that l.c.m. $(m, 2)=2 n$. Thus $m=n$ or $m=2 n$ if $n$ is odd and $m=2 n$ if $n$ is even. We note that $\nabla e=c \frac{d z}{2 w} \otimes e$ has infinite differential Galois group if $c \neq 0$. Thus we may omit this case. 
If $\nabla e=\left(c+\frac{w+w_{0}}{z-z_{0}}\right) \frac{d z}{2 w} \otimes e$ has a cyclic differential Galois group of order $m$, then $\left(z_{0}, w_{0}\right)$ is a point of exact order $m$. Moreover, for a point $\left(z_{0}, w_{0}\right)$ of exact order $m$, there is precisely one value of $c$ such that the differential Galois group is cyclic of order $m$.

We continue the discussion in Section 3 and analyze the exact sequence

$$
0 \rightarrow \mathbb{C} \rightarrow \operatorname{Hom}\left(\pi_{1}(E), \mathbb{C}^{*}\right) \rightarrow E \rightarrow 0
$$

As before, $E=\mathbb{C} /(\mathbb{Z}+\mathbb{C} \tau)$ and we choose as generators $a, b$ of $\pi_{1}(E)$ the circles $\mathbb{R} / \mathbb{Z}$ and $\mathbb{R} / \mathbb{Z} \tau$. This identifies $\operatorname{Hom}\left(\pi_{1}(E), \mathbb{C}^{*}\right)$ with $\mathbb{C}^{*} \times \mathbb{C}^{*}$, by $h \mapsto(h(a), h(b))$. The first map of the exact sequence is $d \mapsto\left(e^{d}, e^{d \tau}\right)$. Put $S^{1}=\left\{z \in \mathbb{C}^{*}|| z \mid=1\right\}$. Every element of $\mathbb{C}^{*} \times \mathbb{C}^{*}$ can be written uniquely as $\left(e^{d}, e^{d \tau}\right) \cdot\left(s_{1}, s_{2}\right)$ with $d \in \mathbb{C}$ and $s_{1}, s_{2} \in S^{1}$. In particular, the restriction of the second map of the exact sequence to $S^{1} \times S^{1}$ is a bijection. Thus for a given point $\left(z_{0}, w_{0}\right) \in E$ of exact order $m$, there is precisely one value of $c$ such that $\nabla e=\left(c+\frac{w+w_{0}}{z-z_{0}}\right) \frac{d z}{2 w} \otimes e$ has a cyclic differential Galois group of order $m$. We note that it is, a priori, difficult to produce a formula for this $c$. However, F. Beukers has proposed a formula (see $[\mathrm{Be}]$ ).

Now we consider $t$ as a variable and investigate a family of Lamé connections $\nabla(t)$ with fixed differential Galois group $D_{n}^{\mathrm{SL}_{2}}$. The corresponding rank one connection $\nabla e=\left(c(t)+\frac{w+w_{0}(t)}{z-z_{0}(t)}\right) \frac{d z}{2 w}$ has the property that $\left(z_{0}(t), w_{0}(t)\right)$ is a point of order $m$. This point is defined over a suitable algebraic extension of $\mathbb{C}(t)$. The monodromy of this family depends in a continuous way on $t$ and lies in $\operatorname{Hom}\left(\pi_{1}(E),\left\{\zeta \in \mathbb{C}^{*} \mid \zeta^{m}=\right.\right.$ $1\})$. Since this group is finite, the family has constant monodromy.

The corresponding algebraic solution for PVI $\left(\frac{1}{8},-\frac{1}{8}, \frac{1}{8}, \frac{3}{8}\right)$ is $z_{0}-\frac{w_{0}}{c}$ according to Theorem 7.1 and the formula for the $(1,2)$ entry of $\stackrel{c}{U}$. A combination of Picard's result and Okamoto's transformation yield the expression $z_{0}-\frac{z_{0}\left(z_{0}-1\right)\left(z_{0}-t\right)}{z_{0}\left(z_{0}-1\right)-t(t-1) z_{0}^{\prime}}$ for this solution. For the uniquely determined $c(t)$ we find therefore the following formula

$$
w_{0}(t) c(t)=z_{0}(t)\left(z_{0}(t)-1\right)-t(t-1) z_{0}(t)^{\prime} .
$$

8. The LOCUS FOR $D_{N}^{\mathrm{SL}_{2}}$ WITH $N=2,3,4$

8.1. Division polynomials. In this subsection we recall some facts on torsion points on elliptic curves directly related to the locus for $D_{n}^{\mathrm{SL}_{2}}$. First we follow [Was] (or [Sil], p. 105) in the description of the division polynomials $\psi_{m}$ with $m \geq 1$. Suppose that the elliptic curve $E$ is given in the Weierstrass form $w^{2}=z^{3}+A z+B$.

The points $\neq 1_{E}$ of order dividing $m$ lie on this affine part of the curve $E$. Their number is $m^{2}-1$ (over an algebraically closed field of 
characteristic 0$)$. The $z$-coordinates of these points are the zeros of a polynomial $\psi_{m} \in \mathbb{Z}[A, B][z, w]$ (with defining relation $w^{2}=z^{3}+A z+$ $B)$. For odd $m, \psi_{m} \in \mathbb{Z}[A, B][z]$ and for $m$ even, $\psi_{m} \in w \mathbb{Z}[A, B][z]$. In the latter case one may replace $\psi_{m}$ by $\psi_{m}^{2}$ in order to have a polynomial in $\mathbb{Z}[A, B][z]$. These polynomials are given by the following recurrence relations

$$
\begin{gathered}
\psi_{0}=0, \psi_{1}=1, \psi_{2}=2 w, \psi_{3}=3 z^{4}+6 A z^{2}+12 B z-A^{2}, \\
\psi_{4}=4 w\left(z^{6}+5 A z^{4}+20 B z^{3}-5 A^{2} z^{2}-4 A B z-8 B^{2}-A^{3}\right), \\
\psi_{2 m+1}=\psi_{m+2} \psi_{m}^{3}-\psi_{m-1} \psi_{m+1}^{3} \text { for } m \geq 2 \\
\psi_{2 m}=(2 w)^{-1} \cdot \psi_{m} \cdot\left(\psi_{m+2} \psi_{m-1}^{2}-\psi_{m-2} \psi_{m+1}^{2}\right) .
\end{gathered}
$$

The zero set of $\psi_{m}^{2}$ (or its square free part $\psi_{m}^{*}$ ) is precisely the set of the $z$-coordinates of the of points with order $\neq 1$ and dividing $m$.

We specialize this by replacing $\mathbb{Z}[A, B]$ by the function field $F:=$ $\mathbb{C}(j)$ of the $j$-line. Then $E$ is the 'universal curve' above the $j$-line. The splitting field of $F$ defined by $\psi_{m}^{*}$ is the function field of the modular curve $X(m)$. The ramified Galois covering $j: X(m) \rightarrow \mathbb{P}^{1}$ is known to have group $\mathrm{PSL}_{2}(\mathbb{Z} / m \mathbb{Z})$. It follows that the Galois group $\mathrm{Gal}_{F}$ of $\bar{F} / F$ acts transitively on the points of precise order $m$. Thus the minimal polynomial for the $z$-coordinate of a point of precise order $m$ is given by $\prod_{d \mid m}\left(\psi_{d}^{*}\right)^{\mu(m / d)}$.

Here we are interested in the division polynomials over the field $\mathbb{C}(t)$ which is a Galois extension of $\mathbb{C}(j)$ with Galois group $S_{3}$. The substitution $z \rightarrow z+\frac{t+1}{3}$ brings $z(z-1)(z-t)$ into the Weierstrass form $z^{3}+A(t) z+B(t)$. Put $\phi_{m}=\psi_{m}\left(A(t), B(t), z-\frac{t+1}{3}\right)$. This is the division polynomial for the Legendre family. Let $\phi_{m}^{*}$ denote the square free part of $\phi_{m}$. The $z$-coordinates of the points of precise order $m$ are the zeros of the polynomial $\prod_{d \mid m}\left(\phi_{d}^{*}\right)^{\mu(m / d)}$.

For odd $m$ this polynomial is irreducible. For even $m$ this polynomial has three irreducible factors, which are permuted by the Galois group $S_{3}$ of $\mathbb{C}(t) / \mathbb{C}(j)$. These statements can be deduced from the Galois action of $\operatorname{Gal}_{\mathbb{C}(t)}$ on the group $(\mathbb{Z} / m \mathbb{Z})^{2}$ of all points of order dividing $m$.

We note that $\sigma \in S_{3}$ permutes in fact the three points of ramification and permutes the three finite singular points of the Lamé connections.

8.2. Points of order 2. The points of precise order two on the elliptic curve yield reducible Lamé connections with cyclic differential Galois groups of order 4 . The formulas for these connections are obtained from the formula $\frac{d}{d z}+U^{*}$ of subsection 7.1, specialized with $w_{0}=0, c=0$ and $z_{0}=0,1, t$. Put $\Delta=4 z(z-1)(z-t)$. One finds 
(1) For $z_{0}=0, \frac{d}{d z}+\frac{1}{\Delta}\left[\begin{array}{cc}z^{2}-t & 0 \\ 0 & -z^{2}+t\end{array}\right]$.

(2) For $z_{0}=1, \frac{d}{d z}+\frac{1}{\Delta}\left[\begin{array}{cc}z^{2}-2 z+t & 0 \\ 0 & -z^{2}+2 z-t\end{array}\right]$.

(3) For $z_{0}=t, \frac{d}{d z}+\frac{1}{\Delta}\left[\begin{array}{cc}z^{2}-2 t z+t & 0 \\ 0 & -z^{2}+2 t z-t\end{array}\right]$.

These equations do not correspond to points of the parameter space $P$. They correspond in fact to the three singular points of $\operatorname{Spec}\left(R_{i n v}\right)$.

8.3. The locus for $D_{2}^{\mathrm{SL}_{2}}$ and points of order 4. The equation for the $z$-coordinates of the points of precise order 4 is

$$
\frac{\phi_{4}(z)}{\phi_{2}(z)}=\left(z^{2}-t\right)\left(z^{2}-2 z+t\right)\left(z^{2}-2 t z+t\right) \in C(t)[z] .
$$

The three irreducible factors produce the components for $D_{2}^{\mathrm{SL}_{2}}$. Let $z_{0}$ denote a zero of the polynomial. The formulas $w_{0}^{2}=z_{0}\left(z_{0}-1\right)\left(z_{0}-t\right)$ and $w_{0} c=z_{0}\left(z_{0}-1\right)-t(t-1) \frac{d z_{0}}{d t}$ and the universal family of subsection 7.1 yield explicit formulas for the connection above the three components for $D_{2}^{\mathrm{SL}_{2}}$. Each component is an open subset of the projective line, parametrized by $\lambda:=z_{0}$. This open subset is determined by the condition $t \neq 0,1, \infty$.

1. The first factor is parametrized by $z_{0}=\lambda, t=\lambda^{2}$. One has further $2 w_{0} c=2 t-z_{0}-z_{0} t$. Put $\Delta=8\left(z-\lambda^{2}\right)(z-1) z$. After conjugation of the system by $\left(\begin{array}{cc}1 & 0 \\ 0 & 8 \lambda\end{array}\right)$, one obtains

$$
\frac{1}{\Delta}\left[\begin{array}{cc}
2 z^{2}-(\lambda+1)^{2} z+\lambda\left(\lambda^{2}+1\right) & (\lambda-1)^{2}(\lambda+1)^{2}(-\lambda-z) \\
\lambda-3 z & -2 z^{2}+(\lambda+1)^{2} z-\lambda\left(\lambda^{2}+1\right)
\end{array}\right]
$$

Using the remarks at the end of Section 1 we get that the corresponding Picard-Vessiot extension is given by:

$$
Y^{8}-2 \frac{k_{1}\left(-z^{2}+\left(2-2 \lambda+2 \lambda^{2}\right) z-\lambda^{2}\right) Y^{4}}{(z-1) z\left(-z+\lambda^{2}\right)}+\frac{k_{1}(z-\lambda)^{4}}{z^{2}(z-1)^{2}\left(z-\lambda^{2}\right)^{2}} .
$$

For all non confluent values of $\lambda$ and all non zero values of $k_{1}$ the galois group of this polynomial over $\mathbb{C}(z)$ is the quaternion group $Q_{8}=D_{2}^{\mathrm{SL}_{2}}$ of order 8. The polynomial is not regular and if we specialize the variable $z$ we obtain the group $Q_{8}: 2$ of order 16 for almost all rational values of $z$ as galois group over $\mathbb{Q}$. 2 . The second factor is parametrized by $z_{0}=\lambda, t=-\lambda^{2}+2 \lambda$. One has $2 w_{0} c=-t-z_{0} t+2 z_{0}$. Put 
$\Delta=8\left(z+\lambda^{2}-2 \lambda\right)(z-1) z$. After conjugation of the system by $\left(\begin{array}{cc}\lambda+2 & 0 \\ 0 & 8\end{array}\right)$, one obtains
$\frac{1}{\Delta}\left[\begin{array}{cc}2 z^{2}+\lambda(\lambda-4) z-\lambda^{2}(\lambda-2) & \lambda(\lambda-2)(\lambda-2+z) \\ (\lambda-2)(-\lambda-2+3 z) \lambda & \lambda^{2}(\lambda-2)-\lambda(\lambda-4) z-2 z^{2}\end{array}\right]$.

Using the remarks at the end of Section 1 we get that the corresponding Picard-Vessiot extension is given by:

$$
Y^{8}+2 \frac{k_{1}\left(z^{2}+\left(2 \lambda^{2}-2 \lambda\right) z-\lambda^{2}\right) Y^{4}}{(z-1) z\left(z+\lambda^{2}-2 \lambda\right)}+\frac{k_{1}(z-\lambda)^{4}}{z^{2}(z-1)^{2}\left(z+\lambda^{2}-2 \lambda\right)^{2}} .
$$

3. The third factor is parametrized by $z_{0}=\lambda, t=\frac{\lambda^{2}}{2 \lambda-1}$. One has $2 w_{0} c=2 t z_{0}-t-z_{0}$. Put $\Delta=8(2 \lambda-1) z(z-1)\left(z-\frac{\lambda^{2}}{2 \lambda-1}\right)$. One finds

$$
\begin{gathered}
\frac{1}{\Delta}\left[\begin{array}{cc}
(2 \lambda-1)\left(2 z^{2}-(2 \lambda+1) z+\lambda\right) & 1 / 8 \frac{(4 \lambda-1)((-2 \lambda+1) z+\lambda)}{\lambda} \\
8 \frac{\lambda((-6 \lambda+3) z+\lambda(4 \lambda-1))}{4 \lambda-1} & (1-2 \lambda)\left(2 z^{2}-(2 \lambda+1) z+\lambda\right)
\end{array}\right] . \\
\text { After conjugation with }\left(\begin{array}{cc}
4 \lambda-1 & 0 \\
0 & 8 \lambda
\end{array}\right) \text { one obtains the system } \\
\frac{1}{\Delta}\left[\begin{array}{cc}
(2 \lambda-1)\left(2 z^{2}-(2 \lambda+1) z+\lambda\right) & (-2 \lambda+1) z+\lambda \\
(-6 \lambda+3) z+\lambda(4 \lambda-1) & (1-2 \lambda)\left(2 z^{2}-(2 \lambda+1) z+\lambda\right)
\end{array}\right]
\end{gathered}
$$

Using the remarks at the end of Section 1 we get that the corresponding Picard-Vessiot extension is given by:

$$
Y^{8}-2 \frac{k_{1}\left(-z^{2}+(-2 \lambda+2) z+\lambda^{2}\right) Y^{4}}{(z-1) z\left((1-2 \lambda) z+\lambda^{2}\right)}+\frac{k_{1}(z-\lambda)^{4}}{z^{2}(z-1)^{2}\left(2 \lambda z-z-\lambda^{2}\right)^{2}} .
$$

8.4. The locus for $D_{3}^{\mathrm{SL}_{2}}$ and points of order 3 and 6 . The locus $P_{3}$ is derived from the points of order 3 and 6 . The $z$-coordinates of the points of precise order 3 are given by the irreducible polynomial

$$
\phi_{3}(z)=-3 z^{4}+4 z^{3} t+4 z^{3}-6 z^{2} t+t^{2}
$$

For the points of precise order 6 this is the polynomial $F_{6}:=\frac{\phi_{6}(z)}{\phi_{3}(z) \phi_{2}(z)}$. The latter has three irreducible factors

$$
\begin{gathered}
F_{6, a}=-z^{4}+4 z^{3}-6 z^{2} t+4 z t^{2}-t^{2}, \\
F_{6, b}=z^{4}-4 z^{3} t+6 z^{2} t-4 z t+t^{2} \\
F_{6, c}=z^{4}-6 z^{2} t+4 z t^{2}+4 z t-3 t^{2} .
\end{gathered}
$$


THE LAMÉ FAMILY OF CONNECTIONS ON THE PROJECTIVE LINE 31

These define the four connected components of $P_{3}$. Each one has a rational parametrization. For a zero $z_{0}$ of $\phi_{6}$ one has $w_{0}^{2}=z_{0}\left(z_{0}-\right.$ 1) $\left(z_{0}-t\right)$ and $w_{0} c=z_{0}\left(z_{0}-1\right)-t(t-1) \frac{d z_{0}}{d t}$. We note that the solutions $q$ of PVI attached to these examples happen to have poles that do not correspond to $t \in\{0,1, \infty\}$, but still the system is well defined. Using Section 7.1, one derives a formula for the connection above each of these components.

1. $\phi_{3}\left(z_{0}, t\right)=0$ is parametrized by $z_{0}=\frac{\lambda^{2}}{2 \lambda-1}, \quad t=-\frac{(\lambda-2) \lambda^{3}}{2 \lambda-1}$. Moreover $6 w_{0} c=t-2 z_{0} t-2 z_{0}+3 z_{0}^{2}$. After conjugation by $\left(\begin{array}{cc}1 & 0 \\ 0 & 24 \lambda\end{array}\right)$ the system is

$$
\left[\begin{array}{cc}
\frac{G_{1}}{(2 \lambda-1) \Delta} & \frac{2^{5}(\lambda-2)(\lambda+1)^{2}\left(\left(\lambda^{2}-\lambda+1\right) z+\lambda^{2}(\lambda-2)\right)}{\Delta} \\
\frac{9(\lambda-2)\left((2 \lambda-1) z-\lambda^{2}\right)}{(2 \lambda-1) \Delta} & \frac{-G_{1}}{(2 \lambda-1) \Delta}
\end{array}\right]
$$

where

$$
\begin{gathered}
\Delta=6^{3} z(z-1)\left(z+\frac{(\lambda-2) \lambda^{3}}{2 \lambda-1}\right) \\
G_{1}=(-54+108 \lambda) z^{2}+12\left(\lambda^{2}-4 \lambda+1\right)(\lambda+1)^{2} z-6 \lambda^{2}(\lambda-2)\left(4 \lambda^{2}-\lambda+4\right)
\end{gathered}
$$

Using the remarks at the end of Section 1 we get that the corresponding Picard-Vessiot extension is given by:

$$
\begin{gathered}
Y^{12}-4 \frac{k_{1}^{3 / 2}\left(\left(2 \lambda^{3}-3 \lambda^{2}+3 \lambda-1\right) z-\lambda^{3}\right) Y^{6}}{(-1+2 \lambda)^{2}(z-1) z\left((-1+2 \lambda) z-2 \lambda^{3}+\lambda^{4}\right)} \\
-\frac{k_{1}\left(\lambda^{2}-2 z \lambda+z\right)^{6}}{(-1+2 \lambda)^{6}(z-1)^{3} z^{3}\left(-z+2 z \lambda-2 \lambda^{3}+\lambda^{4}\right)^{3}} .
\end{gathered}
$$

2. The parametrization of $F_{6, a}\left(z_{0}, t\right)=0$ given by $z_{0}=\lambda^{2}, \quad t=$ $-\frac{(\lambda-2) \lambda^{3}}{2 \lambda-1}$. Conjugation with $\left(\begin{array}{cc}\lambda^{3}+6 \lambda-2 & 0 \\ 0 & 18 \lambda\end{array}\right)$ yields the system

$$
\begin{gathered}
{\left[\begin{array}{cc}
\frac{H_{1}}{\Delta} & \frac{Q}{\Delta} \\
\frac{H_{2}}{\Delta} & \frac{-H_{1}}{\Delta}
\end{array}\right], \text { where } \Delta=36(2 \lambda-1) z(z-1)\left(z+\frac{(\lambda-2) \lambda^{3}}{2 \lambda-1}\right),} \\
Q=(2 \lambda-4)(\lambda+1)^{2}\left((2 \lambda-1) z+\lambda^{2}(\lambda-2)\right), \\
H_{1}=(18 \lambda-9) z^{2}+2(2 \lambda-1)\left(2 \lambda^{3}-6 \lambda^{2}-1\right) z-\lambda^{2}(\lambda-2)\left(2 \lambda^{3}+3 \lambda-4\right), \\
H_{2}=(2 \lambda-4)\left((2 \lambda-1)\left(5 \lambda^{2}-2 \lambda+2\right) z-\lambda^{2}\left(\lambda^{3}+6 \lambda-2\right)\right) .
\end{gathered}
$$


Using the remarks at the end of Section 1 we get that the corresponding Picard-Vessiot extension is given by:

$$
\begin{gathered}
Y^{12}-4 \frac{k_{1}^{3 / 2}(\lambda+1)\left(\lambda^{3}-1+z\right) Y^{6}}{(z-2)\left(\lambda^{4}-2 \lambda^{3}+2 \lambda z-2 \lambda-z+1\right)(z-1)} \\
-\frac{k_{1}\left(-z+\lambda^{2}+1\right)^{6}}{(z-2)^{3}\left(\lambda^{4}-2 \lambda^{3}+2 \lambda z-2 \lambda-z+1\right)^{3}(z-1)^{3}} .
\end{gathered}
$$

3. The parametrization of $F_{6, b}\left(z_{0}, t\right)=0$ is $z_{0}=-\frac{\lambda(\lambda-2)}{2 \lambda-1}, t=-\frac{(\lambda-2) \lambda^{3}}{2 \lambda-1}$. After conjugation by $\left(\begin{array}{cc}2 \lambda^{3}-6 \lambda^{2}-1 & 0 \\ 0 & \lambda^{2}\end{array}\right)$ one obtains the system

$$
\left[\begin{array}{cc}
\frac{H_{1}}{\Delta} & \frac{1 / 9(2 \lambda-1)(\lambda-2)(\lambda+1)^{2}(\lambda-z)}{\Delta} \\
\frac{H_{2}}{\Delta} & \frac{-H_{1}}{\Delta}
\end{array}\right]
$$

where

$$
\begin{gathered}
\Delta=36(2 \lambda-1) z(z-1)\left(z+\frac{(\lambda-2) \lambda^{3}}{2 \lambda-1}\right) \\
H_{1}=(18 \lambda-9) z^{2}+2(\lambda-2)\left(\lambda^{3}+6 \lambda-2\right) z+\lambda(\lambda-2)\left(4 \lambda^{3}-3 \lambda^{2}-2\right) \\
H_{2}=(36 \lambda-72)\left(-(2 \lambda-1)\left(2 \lambda^{2}-2 \lambda+5\right) z-\lambda\left(2 \lambda^{3}-6 \lambda^{2}-1\right)\right) .
\end{gathered}
$$

Using the remarks at the end of Section 1 we get that the corresponding Picard-Vessiot extension is given by:

$$
\begin{gathered}
Y^{12}-4 \frac{k_{1}^{3 / 2}(\lambda-2)(\lambda+1)((2 \lambda-1) z-\lambda+2) Y^{6}}{(2 \lambda-1)^{2}\left((2 \lambda-1) z+\lambda^{4}-2 \lambda^{3}\right)(z-1) z} \\
-\frac{k_{1}\left(2 \lambda z-z+\lambda^{2}-2 \lambda\right)^{6}}{(2 \lambda-1)^{6} z^{3}\left(\lambda^{4}-2 \lambda^{3}+2 \lambda z-z\right)^{3}(z-1)^{3}} .
\end{gathered}
$$

4. The parametrization of $F_{6, c}\left(z_{0}, t\right)=0$ is $z_{0}=-\lambda(\lambda-2), t=$ $-\frac{(\lambda-2) \lambda^{3}}{2 \lambda-1}$. After conjugation by $\left(\begin{array}{cc}\left(\lambda^{2}-4 \lambda+1\right)\left(\lambda^{2}-\lambda+1\right) & 0 \\ 0 & 18 \lambda^{2}\end{array}\right)$ one obtains the system

$$
\left[\begin{array}{cc}
\frac{H_{1}}{\Delta} & \frac{2(\lambda+1)^{2}(\lambda-2)\left((-2 \lambda+1) z+\lambda\left(\lambda^{2}-\lambda+1\right)\right)}{\Delta} \\
\frac{H_{2}}{\Delta} & \frac{-H_{1}}{\Delta}
\end{array}\right]
$$


where $\Delta=36(2 \lambda-1)(z-1)\left(z+\frac{(\lambda-2) \lambda^{3}}{2 \lambda-1}\right) z$,

$$
\begin{gathered}
Q=(2 \lambda-4)(\lambda+1)^{2}\left((-2 \lambda+1) z+\lambda\left(\lambda^{2}-\lambda+1\right)\right), \\
H_{1}=(-9+18 \lambda) z^{2}+4(2 \lambda-1)(\lambda-2)(\lambda+1)^{2} z \\
+\lambda(\lambda-2)\left(2 \lambda^{4}-4 \lambda^{3}-3 \lambda^{2}-4 \lambda+2\right),
\end{gathered}
$$

$H_{2}=-(2 \lambda-4)\left((2 \lambda-1)\left(5 \lambda^{2}-8 \lambda+5\right) z+\lambda\left(\lambda^{2}-4 \lambda+1\right)\left(\lambda^{2}-\lambda+1\right)\right)$.

Using the remarks at the end of Section 1 we get that the corresponding Picard-Vessiot extension is given by:

$$
\begin{gathered}
Y^{12}-4 \frac{k_{1}^{3 / 2}(\lambda-2)\left(\lambda^{3}-3 \lambda^{2}+3 \lambda-2+z\right) Y^{6}}{z(z-1)\left(\lambda^{4}-2 \lambda^{3}+2 \lambda(z+1)-2 \lambda-z\right)} \\
-\frac{k_{1}\left(z+\lambda^{2}-2 \lambda\right)^{6}}{\left(\lambda^{4}-2 \lambda^{3}+2 \lambda(z+1)-2 \lambda-z\right)^{3} z^{3}(z-1)^{3}} .
\end{gathered}
$$

8.5. The locus for $D_{4}^{\mathrm{SL}_{2}}$ and points of order 8. The polynomial $\frac{\phi_{8}(z)}{\phi_{4}(z)}$ has three irreducible factors. One of them is $F_{8}(z)=$ $z^{8}-20 t z^{6}+32\left(t+t^{2}\right) z^{5}-\left(16 t+58 t^{2}+16 t^{3}\right) z^{4}+\left(2 t^{2}+32 t^{3}\right) z^{3}-20 t^{3} z^{2}+t^{4}$.

This factor describes one of the three connected components for $D_{4}^{\mathrm{SL}_{2}}$. A rational parametrization of $F_{8}\left(z_{0}, t\right)=0$ is given by

$$
z_{0}=-4 \frac{(\lambda-1)^{3} \lambda}{(2 \lambda-1)^{3}}, \quad t=16 \frac{\lambda^{4}(\lambda-1)^{4}}{(2 \lambda-1)^{4}}
$$

After conjugation with

$$
\left[\begin{array}{cc}
\left(36 \lambda^{4}-32 \lambda^{3}+4 \lambda^{2}+1\right)\left(6 \lambda^{2}-4 \lambda+1\right) & 0 \\
0 & 32(\lambda-1) \lambda^{3}(2 \lambda-1)^{3}
\end{array}\right]
$$

one obtains the system

$$
\left[\begin{array}{cc}
\frac{H_{1}}{\Delta} & \frac{Q}{\Delta} \\
\frac{H_{2}}{\Delta} & \frac{-H_{1}}{\Delta}
\end{array}\right]
$$

where

$$
\begin{gathered}
\Delta=32(2 \lambda-1)^{7} z(z-1)\left(z-16 \frac{\lambda^{4}(\lambda-1)^{4}}{(2 \lambda-1)^{4}}\right) \\
Q=1 / 4\left(2 \lambda^{2}-1\right)^{2}\left(2 \lambda^{2}-4 \lambda+1\right)^{2} \\
\left(-4 \lambda\left(6 \lambda^{2}-4 \lambda+1\right)(\lambda-1)^{3}+\left(2 \lambda^{2}-4 \lambda+3\right)(2 \lambda-1)^{3} z\right) \\
H_{1}=-\left(9-16 \lambda+4 \lambda^{2}+4 \lambda^{4}\right)\left(2 \lambda^{2}-4 \lambda+1\right)^{2}(2 \lambda-1)^{3} z+8(2 \lambda-1)^{7} z^{2} \\
-4 \lambda\left(144 \lambda^{8}-448 \lambda^{7}+608 \lambda^{6}-416 \lambda^{5}+152 \lambda^{4}-48 \lambda^{3}+24 \lambda^{2}-8 \lambda+1\right)(\lambda-1)^{3}
\end{gathered}
$$




$$
\begin{gathered}
H_{2}=16 \lambda\left(36 \lambda^{4}-32 \lambda^{3}+4 \lambda^{2}+1\right)\left(6 \lambda^{2}-4 \lambda+1\right)(\lambda-1)^{3} \\
+12\left(40 \lambda^{6}-80 \lambda^{5}+100 \lambda^{4}-112 \lambda^{3}+86 \lambda^{2}-36 \lambda+7\right)(2 \lambda-1)^{3} z
\end{gathered}
$$

8.6. Decidable. Let a differential operator $\frac{d}{d z}+\sum_{j=1}^{3} \frac{A_{i}}{z-s_{j}}$ be given where the $A_{j}$ satisfy the conditions of Section 5 . We suppose that the equation is irreducible and that the field $K$, generated by all entries (including $t$ ), is a finite extension of $\mathbb{Q}(t)$. We claim that there is an algorithm deciding whether the differential Galois group is $D_{n}^{\mathrm{Sl}_{2}}$ for some integer $n \geq 2$ or equals $D_{\infty}^{\mathrm{Sl}_{2}}$. This claim is equivalent to the assertion that there is an algorithm deciding whether a point on the elliptic curve $w^{2}=c z(z-1)(z-t)$ has finite order or not. This problem has been studied by B. Dwork, F. Baldassarri and B.M. Trager et al.(see [Tra]).

If $K$ is a number field, then according to a theorem of L. Merel [Mer], there is an effective function $N$ of $[K: \mathbb{Q}]$ such that any elliptic curve, defined over $K$, has, over $K$, no point of order $>N$. Hence there is an algorithm in this situation.

Suppose that $t$ is transcendent. Then there is also an effective bound on the order $n$ of points on $E$ with values in $K$. This bound comes from the fact that the genera of the modular curves $X_{1}(n)$ tend to infinity. If the genus of $X_{1}(n)$ is larger than the genus of $K$ (viewed as a curve) then the elliptic curve has no points of order $n$ with values in $K$. Again there is an algorithm in this case.

\section{OKAMoto MOdUli SPACE}

We supplement our construction of moduli spaces with the description of Okamoto's one in the special case of Lamé connections, that is the space of initial conditions of Painlevé VI equation with parameters $\theta_{i}=\frac{1}{2}$, i.e. $(\alpha, \beta, \gamma, \delta)=\left(\frac{1}{8},-\frac{1}{8}, \frac{1}{8}, \frac{3}{8}\right)$, following [15] (see also [20] or [9]). We consider the Zariski open subset of Lamé systems

$$
\frac{d}{d z}+\frac{A_{1}}{z}+\frac{A_{2}}{z-1}+\frac{A_{3}}{z-t} \quad \text { with } \quad A_{1}+A_{2}+A_{3}=\left(\begin{array}{cc}
\frac{1}{4} & 0 \\
0 & -\frac{1}{4}
\end{array}\right) .
$$

As in Section 5 we exclude those for which the vector $\left(\begin{array}{l}0 \\ 1\end{array}\right)$ is invariant (i.e., some $\left.b_{i} \neq 0\right)$. We define new variables

$$
p=\frac{\frac{1}{4}-a_{1}}{q}+\frac{\frac{1}{4}-a_{2}}{q-1}+\frac{a_{1}+a_{2}}{q-t} \quad \text { and } \quad q=-t \frac{a_{1}^{2}-\frac{1}{16}}{(t-1) H-t\left(a_{1}^{2}-\frac{1}{16}\right)} .
$$


Now, consider the 3 -dimensional variety $M:=\left(\mathbf{P}^{1}-\{0,1, \infty\}\right) \times \mathcal{O}(-2)$ where $\mathcal{O}(-2)$ denotes the total space of the degree -2 line bundle on $\mathbf{P}^{1}: M$ is defined by charts $(t, x, y) \in\left(\mathbf{P}^{1}-\{0,1, \infty\}\right) \times \mathbb{C}^{2}$ and $(\tilde{t}, \tilde{x}, \tilde{y}) \in\left(\mathbf{P}^{1}-\{0,1, \infty\}\right) \times \mathbb{C}^{2}$ with transition map

$$
(t, x, y) \mapsto(\tilde{t}, \tilde{x}, \tilde{y})=\left(t, \frac{1}{x}, \frac{y}{x^{2}}\right)
$$

Consider in $M$ the following 8 disjoint lines

$$
\begin{array}{cl}
l_{0}:\{x=0, y=0\} & l_{0}^{\prime}:\left\{x=0, y=\frac{t}{2}\right\} \quad l_{1}:\{x=1, y=0\} \\
l_{1}^{\prime}:\left\{x=1, y=\frac{1-t}{2}\right\} & l_{t}:\{x=t, y=0\} \quad l_{t}^{\prime}:\left\{x=t, y=\frac{t(t-1)}{2}\right\} \\
l_{\infty}:\{\tilde{x}=0, \tilde{y}=0\} \quad l_{\infty}^{\prime}:\left\{\tilde{x}=0, \tilde{y}=\frac{1}{2}\right\}
\end{array}
$$

Consider also the divisor $Z \subset M$ which is the union of

$$
Z_{0}=\{x=0\}, \quad Z_{1}=\{x=1\}, \quad Z_{t}=\{x=t\}, \quad Z_{\infty}=\{\tilde{x}=0\} .
$$

Denote by $\widetilde{M}$ the blowing-up of $M$ with centers the 8 lines above and $\widetilde{Z} \subset \widetilde{M}$ the strict transform of $Z$. Then, Okamoto moduli space is $\mathcal{M}:=\widetilde{M} \backslash \widetilde{Z}$. In fact, if $\Theta$ denotes the restriction to $\mathcal{M}$ of the exceptional divisor over $l_{\infty}$, then $\mathcal{M}-\Theta$ is the moduli space of the family of systems described above under diagonal conjugacies. The map from our moduli space $P$ computed in Section 5 to Okamoto's one is given by

$$
P-\rightarrow \mathcal{M} \backslash \Theta ;\left(t, a_{1}, a_{2}, H\right) \mapsto(x, y)=(t, q, q(q-1)(q-t) p) .
$$

In fact, the upper triangular families are defined in the first chart by

$$
\left\{y=\frac{1}{2}(x-1)(x-t)\right\}, \quad\left\{y=\frac{1}{2} x(x-1)\right\} \quad \text { and } \quad\left\{y=\frac{1}{2} x(x-t)\right\}
$$

(or we should say their strict transform in $\mathcal{M}$ ). The smooth part of $P$ is sent isomorphically to the complement of those 3 rational surfaces in $\mathcal{M} \backslash \Theta$ by the above map. Now, one can check that $\Theta$ is our special family. By the way, the total space $\mathcal{M}$ is the moduli space of all Lamé connections (with varying vector bundle) after omitting a very reducible part.

\section{REFERENCES}

[1] W. Barth and J. Michel - Modular curves and Poncelet polygons - Math. Ann. 295 (1993), 25-49.

[2] M. Berkenbosch and M. van der Put - Families of linear differential equations on the projective line - to appear 2006.

[3] F. Beukers -Solutions of a Lamé system - private notes, November 10, 2004. 
[4] F. Beukers and A. van der Waall - Lamé equations with algebraic solutions - J. Differential Equations 197 (2004), 1-25.

[5] B. Chiarellotto - On Lamé Operators which are Pullbacks of Hypergeometric Ones - Trans. Amer. Math. Soc., Vol 347 (1995), 2735-2780.

[6] N.J. Hitchin - Poncelet Polygons and the Painlevé Equations - Geometry and analysis (Bombay, 1992), 151-185, Tata Inst. Fund. Res., Bombay 1995.

[7] N.J. Hitchin - Twistor spaces, Einstein metrics and isomonodromic transformations - J. Differential Geometry 42 (1995), 30-112.

[8] M. van Hoeij and M. van der Put - Descent for differential modules and skew fields - preprint 2003.

[9] M. Inaba, K. Iwasaki and M. Saito - Dynamics of the Sixth Painlevé equation math.AG/0501007.

[10] M. Jimbo and T. Miwa - Monodromy preserving deformations of linear ordinary differential equations with rational coefficients II - Physica 2D (1981a), 407-448.

[11] S.Lang - Elliptic curves: Diophantine analysis - Grundlehren no 231, 1978.

[12] Yu.I. Manin - Sixth Painlevé equation, universal elliptic curve, and Mirror of $\mathbb{P}^{2}$ - AMS Transl. (2) 186 (1998), 131-151.

[13] M. Mazzocco - Picard and Chazy solutions to the Painlevé VI equation - Math. Ann. 321 no 1, (2001), 157-195.

[14] L. Merel - Bornes pour la torsion des courbes elliptiques sur les corps de nombres - Invent. Math., 124 (1996), 437-449.

[15] K. Okamoto, Sur les feuilletages associés aux équations du second ordre à points critiques fixes de P. Painlevé - Japan J. Math. 5 (1979), 1-79.

[16] K. Okamoto, Studies on the Painlevé equations I. Sixth Painlevé equation $P_{\mathrm{VI}}$, Ann. Mat. Pura Appl. (4) 146 (1987), 337-381.

[17] É. Picard - Mémoire sur la théorie des fonctions algébriques de deux variables - Journal de Mathématiques Pures et Appliquées 5 (1889), 135-319.

[18] M.-H. Saito and T. Takebe - Classification of Okamoto-Painlevé pairs - Kobe J. Math., 19 (2002), 21-50.

[19] M.-H. Saito and H. Terajima - Nodal curves and Riccati solutions of Painlevé equations - J. Math. Kyoto Univ., 44-3 (2004), 529-568.

[20] H. Sakai - Rational surfaces associated with affine root systems and geometry of the Painlevé equations - Comm. Math. Phys. 220 (2001), 165-229.

[21] J.H. Silverman - The Arithmetic of Elliptic Curves - GTM 106, Springer Verlag 1986.

[22] M.F. Singer - Moduli of Linear Differential Equations on the Riemann Sphere - Pac.J. Math., 160, (1993), 343-395.

[23] B.M. Trager - Integration of algebraic functions - Ph.D. Thesis M.I.T., September 1984.

[24] L. C. Washington - Elliptic Curves - Chapman-Hall 2003. 
F. Loray (C.N.R.S.) And F. Ulmer - IRMAR, Université de Rennes 1, Campus de Beaulieu, 35042 Rennes Cedex, France

M. van der Put - Department of mathematics, University of GroninGen, P.O.Box 800, 9700 AV, Groningen, The Netherlands

E-mail address: frank.loray@univ-rennes1.fr, mvdput@math.rug.nl and felix.ulmer@univ-rennes1.fr 